\title{
Targeting castration-resistant prostate cancer with androgen receptor antisense oligonucleotide therapy
}

\author{
Marco A. De Velasco, ${ }^{1,2}$ Yurie Kura, ${ }^{1}$ Kazuko Sakai, ${ }^{2}$ Yuji Hatanaka, ${ }^{1}$ Barry R. Davies, ${ }^{3}$ \\ Hayley Campbell, ${ }^{3}$ Stephanie Klein, ${ }^{3}$ Youngsoo Kim, ${ }^{4}$ A. Robert MacLeod, ${ }^{4}$ Koichi Sugimoto, \\ Kazuhiro Yoshikawa, ${ }^{5}$ Kazuto Nishio, ${ }^{2}$ and Hirotsugu Uemura ${ }^{1}$ \\ 'Department of Urology and 'Department of Genome Biology, Kindai University Faculty of Medicine, Osakasayama, \\ Osaka, Japan. ${ }^{3}$ Oncology, IMED Biotech Unit, AstraZeneca, Cambridge, United Kingdom. ${ }^{4}$ Department of Antisense Drug \\ Discovery, Ionis Pharmaceuticals, Carlsbad, California, USA. ${ }^{5}$ Research Creation Support Center, Aichi Medical University, \\ Nagakute, Aichi, Japan.
}

\begin{abstract}
Sustained therapeutic responses from traditional and next-generation antiandrogen therapies remain elusive in clinical practice due to inherent and/or acquired resistance resulting in persistent androgen receptor (AR) activity. Antisense oligonucleotides (ASO) have the ability to block target gene expression and associated protein products and provide an alternate treatment strategy for castration-resistant prostate cancer (CRPC). We demonstrate the efficacy and therapeutic potential of this approach with a Generation-2.5 ASO targeting the mouse AR in genetically engineered models of prostate cancer. Furthermore, reciprocal feedback between AR and PI3K/AKT signaling was circumvented using a combination approach of AR-ASO therapy with the potent pan-AKT inhibitor, AZD5363. This treatment strategy effectively improved treatment responses and prolonged survival in a clinically relevant mouse model of advanced CRPC. Thus, our data provide preclinical evidence to support a combination strategy of next-generation ASOs targeting AR in combination with AKT inhibition as a potentially beneficial treatment approach for CRPC.
\end{abstract}

Conflict of interest: MAD and HU received funding support from AstraZeneca. BRD, HC, and SK are employees of AstraZeneca. YK and ARM are employees of lonis Pharmaceutical.

Copyright: (c) 2019 American Society for Clinical Investigation

Submitted: June 5, 2018 Accepted: August 1, 2019 Published: August 6, 2019.

Reference information: /CI Insight. 2019;4(17):e122688. https://doi. org/10.1172/jici.insight.122688

\section{Introduction}

Androgen receptor (AR) signaling plays important roles during normal prostate development and homeostasis, and it contributes to prostate tumorigenesis and disease progression (1). Consequently, the AR signaling pathway is an important axis for the treatment of advanced prostate cancer ( $\mathrm{PCa}$ ), and androgen deprivation therapy (ADT) continues to be the main palliative treatment for men with locally advanced or metastatic disease. ADT by surgical or chemical castration is ultimately rendered ineffective, yielding to incurable castration-resistant PCa (CRPC). Potentially novel pharmacological approaches aiming to circumvent castration resistance have focused on hindering AR transcriptional activity by blocking ligand binding and disrupting its translocation to the nucleus (enzalutamide) or by inhibiting steroidogenesis through the inhibition of CYP17A1 (abiraterone) $(2,3)$. The second-generation antiandrogens enzalutamide and abiraterone have significantly improved survival over traditional hormonal and other nonsteroidal therapies; however, many patients still fail to respond or continue to develop secondary resistance (4-8).

CRPC is characterized by the persistent activation of AR, despite low levels of circulating androgens. Continued AR activity has been implicated with ligand-dependent (e.g., AR overexpression, gene amplification) and ligand-independent (e.g., expression of splice variants that lack the LBD) resistance mechanisms (9-11). Regardless of the underlying mechanisms driving CRPC resistance, all depend on AR protein expression; thus, a therapeutic strategy that silences $A R$ gene expression is a potentially effective approach to treat CRPC. Antisense oligonucleotides (ASOs) can intrinsically block specific gene targets, preventing the synthesis of their associated proteins, and have thus become an alternative treatment approach for various human diseases. ASOs offer several qualities that make them attractive as an alternate anticancer strategy; however, lack of efficacy due to poor bioavailability and suboptimal target engagement has limited their therapeutic potential. Recent advances in ASO formulations have greatly improved the effectiveness of systemic ASO delivery. Generation-2.5 ASOs are a current class of potent antisense molecules that 
incorporate a 3-10-3 (S)-constrained ethyl gapmer with a phosphorothioate (cEt) backbone modification that greatly improves potency and biodistribution (12). Preclinical research has shown that Generation-2.5 ASOs targeting human $A R$ are capable of effectively suppressing the expression of full-length AR (AR-FL) and its splice variants, resulting in antitumor activity in models of enzalutamide-resistant CRPC (13).

$\mathrm{PCa}$, however, develops through a complex multistep process that includes several genomic and nongenomic alterations besides AR. The PI3K/protein kinase B (AKT)/mTOR pathway is a key signal pathway involved in regulating numerous cellular processes, and its dysregulation is implicated in various cancers (14). The PI3K/AKT pathway is highly conserved and is negatively regulated by the phosphatase and tensin homolog (PTEN) tumor suppressor (15). In PCa, PI3K/AKT is frequently upregulated as a result of biallelic loss of PTEN, activating mutations in $A K T 1$ and $P I K 3 C A / B$, and overexpressed growth factors (16-21). In mice, inactivation of Pten in the prostate drives the stage-specific development of $\mathrm{PCa}$ (22). Complex interactions between AR and PI3K/AKT pathways have been reported and are likely to contribute to enhancing cancer cell survival after ADT and promote therapeutic escape to PI3K/AKT-targeted therapies $(23,24)$.

Herein, we characterize and describe the in vivo activity of a Generation-2.5 ASO targeting mouse $A r$ in an established genetically engineered mouse (GEM) model of PCa $(25,26)$. Our studies also show the therapeutic potential of ASO therapy in models of castration-naive PCa (CNPC) and CRPC. Lastly, we show that a therapeutic strategy of combined $A r$-ASO blockade and AKT inhibition can augment antitumor responses and promote longer survival in a GEM model of advanced CRPC.

\section{Results}

Pharmacodynamic characterization of a Generation-2.5 ASO targeting mouse Ar in Pten-deficient prostate tumors. We used an established GEM model of Pten-deficient PCa (25) to characterize the biodistribution and potency of ISIS581088, a Generation-2.5 ASO targeting intron 1 of mouse Ar. We first examined the uptake of ISIS581088 and scrambled control ASO (Ctrl-ASO) by prostate tumors after systemic delivery in a 96-hour time course (Figure 1A) and performed IHC using an antibody that detects the phosphorothiaoate backbone of the Generation-2.5 ASOs to localize the ASO in neoplastic epithelial cancer cells (27). Distribution of ISIS581088 in mouse prostatic intraepithelial neoplasia (mPIN) lesions was observed as early as 2 hours after systemic administration (Figure 1B). Uptake of the ASOs was greater in high-grade mPIN lesions - particularly in those of the ventral lobe, which had the higher concentration of high-grade mPIN. ASO uptake was less in nondysplastic prostate glands and normal surrounding tissues but was readily incorporated by macrophages (Supplemental Table 1; supplemental material available online with this article; https://doi. org/10.1172/jci.insight.122688DS1). We next sought to determine the efficacy of ISIS581088 by evaluating its ability to silence $A r$ mRNA and AR protein expression. ISIS581088 strongly inhibited $A r$ mRNA 24 hours after a single dose, remained repressed at day 4 with daily dosing (Figure 1C), and was consistent with decreased AR protein expression in cancer cells (Figure 1D).

Chemical modifications can extend the half-life of therapeutic ASOs, requiring lower dosing; thus, we further evaluated the pharmacodynamic activity of ISIS581088 on Ar mRNA, AR protein, and AR target genes $F k b p 5$, Nkx3.1, and Msmb in matched samples using a treatment schedule consisting of a loading phase (daily administration of the ASOs for 5 days), followed by a maintenance phase of intermittent dosing (Supplemental Figure 1A). Treatment with ISIS581088 led to 50\% reductions of Ar mRNA and AR protein levels vs. Ctrl-ASO at 24 and 72 hours, respectively (Figure $1 \mathrm{E}$ and Supplemental Figure 1, B-D). There was a strong correlation between the expression levels of $A r$ mRNA, AR protein, and AR target genes in ISIS581088-treated mice (Figure 1F). Expression levels of Fkbp5, Nkx3.1, and Msmb decreased after treatment with ISIS581088 and reached nadir levels at day 11 (Supplemental Figure 1E). Overall, our data show that persistent ASO-mediated silencing of $A r$ is necessary to suppress AR transcriptional activity.

ISIS581088 suppresses putative AR splice variants. Aberrant AR protein resulting from alternatively spliced transcripts or mutations has been implicated in castration resistance and therapeutic escape to second-generation antiandrogen therapies $(7,8,28)$. ASOs can cause the breakdown of target mRNA, resulting in loss of protein translation. Since ISIS581088 targets intron 1 of mouse $A r$, it could potentially suppress all AR protein products, including those that result from alternative splicing. We tested this concept by examining the capability of ISIS581088 to suppress aberrantly expressed AR protein products in our mouse PCa model, since functionally active AR splice variants (AR-Vs) have been described in other mouse PCa models $(29,30)$. Many of the clinically relevant AR-Vs are truncated and lack the hinge and ligand binding domain (LBD) 
A

\begin{tabular}{|c|c|c|c|c|c|c|}
\hline \multirow{2}{*}{\multicolumn{2}{|c|}{$P S A^{\text {cre }}: P T E N^{f f f f}$}} & \multicolumn{5}{|c|}{ ISIS581088 or Ctrl ASO } \\
\hline & & & $\downarrow$ & $\downarrow$ & $\downarrow$ & \} $\\
{\hline} &{024} &{8} &{24} &{48} &{72} &{96(\mathrm{~h}} \\
{\hline} &{\text { 【】 }} &{\text { I }} &{\downarrow} &{\downarrow} &{\text { t }} &{\text { I }} \\
{\hline \begin{array}{l}\text { IS581 } \\
\text { Ctrl A }\end{array}} &{\begin{array}{l}\checkmark \checkmark \checkmark \\
\checkmark\end{array}} &{\checkmark} &{\text { 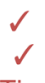 }} &{\checkmark} &{\checkmark} &{ } \\
$\hline
\end{tabular}

C

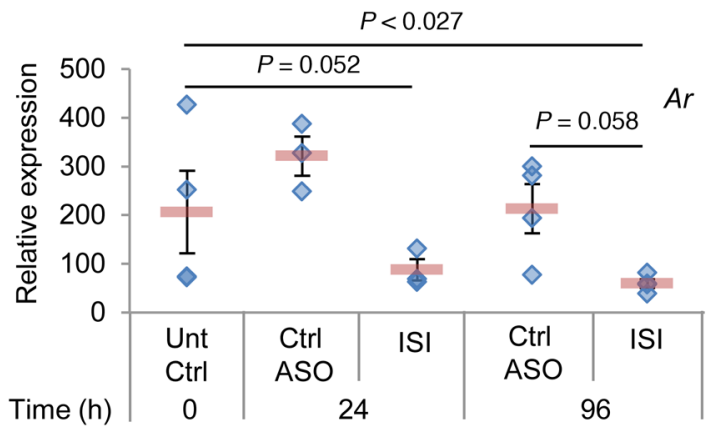

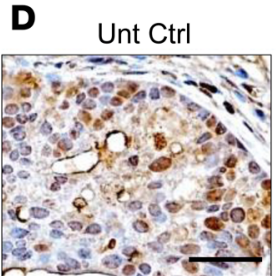

$\mathrm{Oh}$

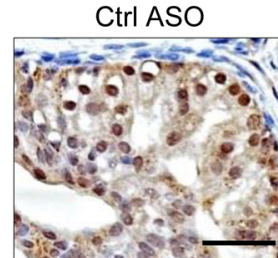

$96 \mathrm{~h}$

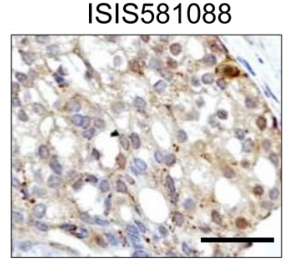

$96 \mathrm{~h}$
E

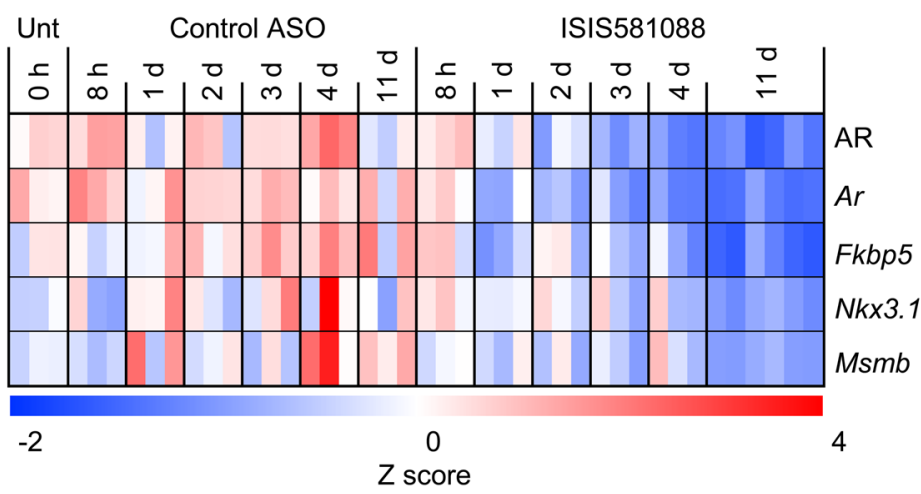

B

\begin{tabular}{|c|c|c|c|c|c|c|c|c|c|c|c|c|}
\hline \multicolumn{2}{|c|}{ mPIN } & \multicolumn{3}{|c|}{ Ctrl ASO } & \multicolumn{8}{|c|}{ ISIS581088 } \\
\hline \multirow{4}{*}{$\mathrm{DP}$} & 1 & - & $-1+$ & + & - & - & - & - & - & - & - & + \\
\hline & 2 & - & $-1+$ & + & - & $-1+$ & $-1+$ & $-1+$ & + & - & + & + \\
\hline & 3 & - & $-1+$ & ++ & - & + & + & + & & ++ & ++ & ++ \\
\hline & 4 & & & & & & - & ++ & & & & \\
\hline \multirow{4}{*}{ VP } & 1 & - & $-/+$ & + & - & $-1+$ & $-1+$ & $-1+$ & - & & - & + \\
\hline & 2 & - & + & ++ & - & $-1+$ & + & + & $-1+$ & $-1+$ & $-1+$ & ++ \\
\hline & 3 & - & + & ++ & - & ++ & ++ & ++ & ++ & ++ & ++ & ++ \\
\hline & 4 & - & $-/+$ & ++ & - & ++ & ++ & ++ & ++ & ++ & ++ & \\
\hline \multicolumn{2}{|c|}{ Time (h) } & 0 & 24 & 96 & 0 & 2 & 4 & 8 & 24 & 48 & 72 & 96 \\
\hline & & & & & & & \multicolumn{5}{|c|}{ IHC distribution score } & \\
\hline & & & & & & & $\mathrm{np}$ & - & $-1+$ & + & ++ & \\
\hline
\end{tabular}
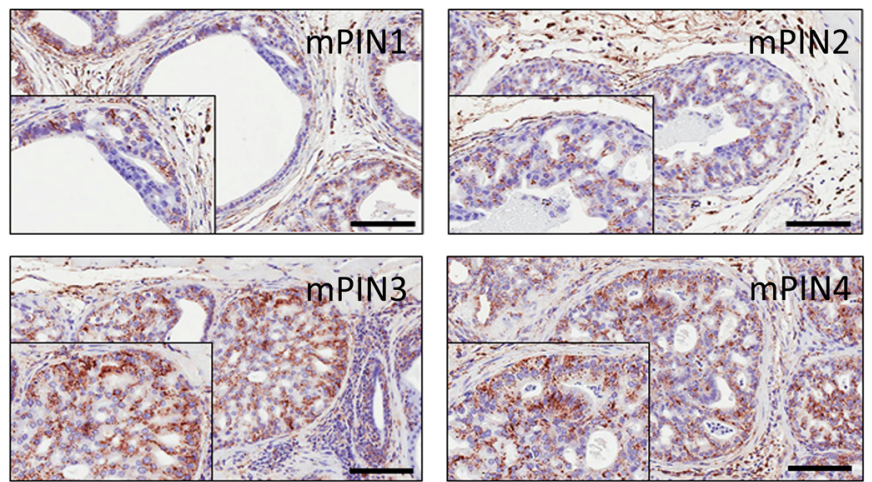

$\mathbf{F}$

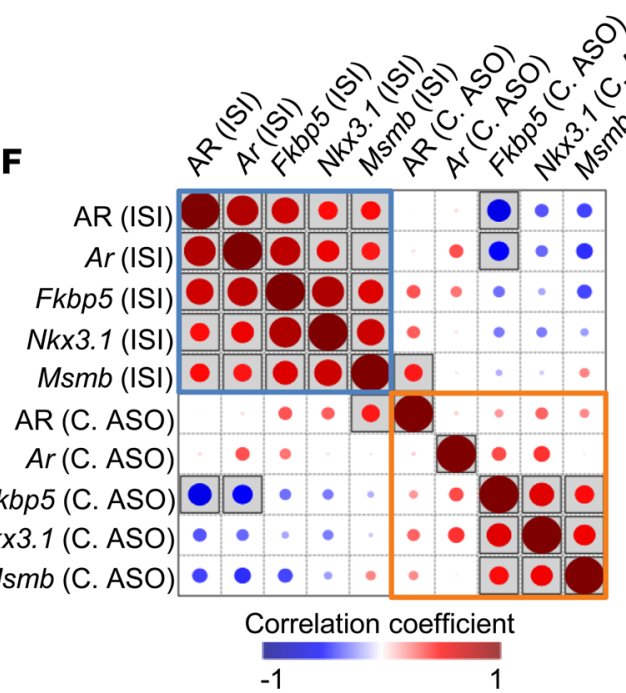

Figure 1. Pharmacodynamic activity of ISIS581088 in mouse prostate tumors. (A) Conditional Pten-KO (18-19 weeks of age, $n=3-4$ mice/group) mice received ISIS581088 (40 mg/kg i.p.) or the control ASO (40 mg/kg i.p.) as indicated. (B) Semiquantitative analysis and representative IHC images of ASO uptake in mPIN lesions of the dorsal (DP) and ventral (VP) lobes of mouse prostate. Cumulative distribution of the ASO was assessed according to distribution patterns against an antibody targeting the Generation-2.5 ASO backbone (np, not present; -, negative; +/-, slight; +, minimal; ++, moderate; $n=2-4$ mice/group) Scale bars: $100 \mu \mathrm{m}$. (C) Ar mRNA expression analysis by qPCR. Horizontal bars represent \pm SEM, and diamonds represent individual samples. Significance represent Student-Newman-Keuls post hoc test for individual comparisons, upon significant 1-way ANOVA $\left(F_{4,22}=5.301, P=0.005\right)$. (D) AR protein expression by IHC. Scale bar: $50 \mu \mathrm{m}$. (E) Heatmap of AR protein, Ar mRNA, and AR target gene expression by qPCR in 20 -week-old Pten-KO treated with ISIS581088 (ISI) or control ASO (Ctrl ASO) ( $n=3-6$ mice/group). (F) Correlation matrix of AR protein, Ar mRNA, and AR target gene expression; shaded squares represent $P<0.05$

located in exons 5-8 (31). We performed capillary protein electrophoresis (Simple Western Assay) in normal WT-Pten prostate tissue and Pten-deficient PCa using AR antibodies targeting the N-terminal domain (NTD) and the LBD to determine the presence of aberrant AR. Presumably, the antibody targeting the NTD would detect AR-FL and truncated AR, whereas the AR antibody targeting the LBD would only detect AR-FL (Supplemental Figure 3C). Consistent with the other reports, Pten-deficient tumors exhibited additional AR products, including a band with a molecular weight of $75-80 \mathrm{kDa}$, which corresponds to the size of a previously 
reported mouse functional AR-V (Figure 2, A and B; Supplemental Figure 2; and refs. 29, 30). Moreover, we noted observed greater expression of high molecular weight bands at $180 \mathrm{kDa}$ and $230-240 \mathrm{kDa}$. Notably, these putative mouse AR-Vs and aberrant AR bands remained present after surgical castration and were constitutively expressed even at 60 days after androgen withdrawal (Supplemental Figure 3, A and B). To confirm the presence of putative AR-Vs in our model, we examined the IHC expression patterns between antibodies specific for the NTD of AR (detecting AR-FL and AR-Vs) and those of an AR antibody targeting the serine 650 hinge region of AR (AR-S650, AR-FL) (Supplemental Figure 3C). Expression patterns between the 2 antibodies were similar in normal prostate glands (Supplemental Figure 3D); however, these became varied in castration-naive prostate tumors, and expression of AR-S650 became more discordant after surgical castration, especially in castration-resistant tumors (Supplemental Figure 3E). Together, these data provide evidence to support a role of aberrant $\mathrm{AR}$ in prostate tumorigenesis and progression after androgen withdrawal.

We next determined if knockdown of $A r$ using ISI581088 would suppress the expression of both AR-FL and aberrant AR (putative AR-Vs and high-molecular weight AR) in mouse prostate tumors. Given our results in Supplemental Figure 3, A and B, the highest ratio of aberrant AR/AR-FL occurred 7-14 days after orchidectomy; therefore, we next examined the effect of $A r$-ASO silencing and the expression of AR-FL and aberrant AR in prostate tumors 10 days after treatment with ISIS581088 and compared it with the effects of surgical castration. Immunoblot analysis showed that silencing $A r$ with ISIS581088 moderately reduced the expression levels of AR-FL and aberrant AR (Figure 2C). However, quantitative PCR (qPCR) analysis showed potent inhibition of $A r$ mRNA at exons 2 and 7 (Figure 2D), suggesting that some proteins expressed in the immunoblots Figure $2 \mathrm{C}$ may be remnants of pretreatment AR. Additionally, AR-V proteins have been shown to have to have a longer half-life compared with AR-FL (32). To test this notion, we evaluated AR-FL and AR-V expression after 4 weeks of treatment with ISIS581088, and indeed, longer exposure to the ASO potently downregulated all forms of AR protein in tumors (Figure 2, $\mathrm{A}$ and B, and Supplemental Figure 2). These results show that silencing Argene expression with a Generation-2.5 Ar-ASO, which binds to intron 1, can target all forms of AR and, in principle, has the potential to overcome mechanisms of resistance manifested by aberrant AR.

Preclinical efficacy of Generation-2.5 Ar-ASO in Pten-deficient prostate tumors. We next observed the antitumor activity of Generation-2.5 Ar-ASO therapy with ISIS581088 compared with surgical castration in the Pten-deficient prostate tumor model, as shown in Figure 3A. Mice were evaluated after 4 weeks of treatment, and no significant changes in mouse body weights were observed between the groups (Supplemental Figure 3). Treatments with ISIS581088 and orchidectomy significantly reduced the size and weight of the genitourinary tract (GUT) by $55.6 \%$ and $74.2 \%$ of control vehicle, respectively (Figure 3, B and C). The mouse GUT includes prostate tumor tissue, as well as normal androgen-responsive accessory sex organs; therefore, to more precisely determine the effects of the treatments on tumor tissues, we used tumor area as surrogate for tumor burden. Treatments with both ISIS581088 and orchidectomy significantly reduced tumor area by $34.5 \%$ and $49.7 \%$, respectively (Figure 3, B and D). Histologically, mice treated with ISIS581088 exhibited similar changes to orchidectomized mice. Both treatments reduced the numbers of and severity of neoplastic lesions, increased glandular atrophy and apoptotic bodies, and accumulated cellular debris in glandular lumens (Figure 3E). The prostate glands were a third smaller in mice after ISIS581088 treatment as compared with orchidectomy, resulting in an increased thickness of the stromal compartment compared with glandular structures. No histological changes were noted between mice treated with vehicle and the Ctrl-ASO (Figure 3E). The pharmacological activity of ISIS581088 was confirmed with the suppression of $A r$ mRNA and resulted in reduced levels of AR protein (Figure 3, F-H). Treatments with ISIS581088 and surgical castration resulted in potent antiproliferative activity indicated by a reduction in KI67-positive epithelial cancer cells; remarkably, suppression of AR by ISIS581088 had a stronger antiproliferative effect than surgical castration ( $48.5 \%$ vs. $66.7 \%$ of control vehicle, Figure $3 \mathrm{G})$. Although both treatments significantly induced apoptosis, orchidectomy produced a 1.4-fold higher number of cleaved caspase-3-labeled cells than ISIS581088 (Figure 3, G and H). These data show that systemic administration of Generation-2.5 $A r$-ASO is capable of $A r$ silencing, resulting in potent antitumor activity comparable with that of surgical castration.

Ar knockdown with ISIS581088 produces greater antitumor activity compared with other pharmacological antiandrogen therapies. We next compared Generation-2.5 Ar-ASO therapy with different antiandrogen treatments, including surgical castration, nonsteroidal antiandrogens using enzalutamide and apalutamide (ARN-509, which is structurally and functionally similar to enzalutamide), the steroidal CYP17A1 inhibitor abiraterone, 
A

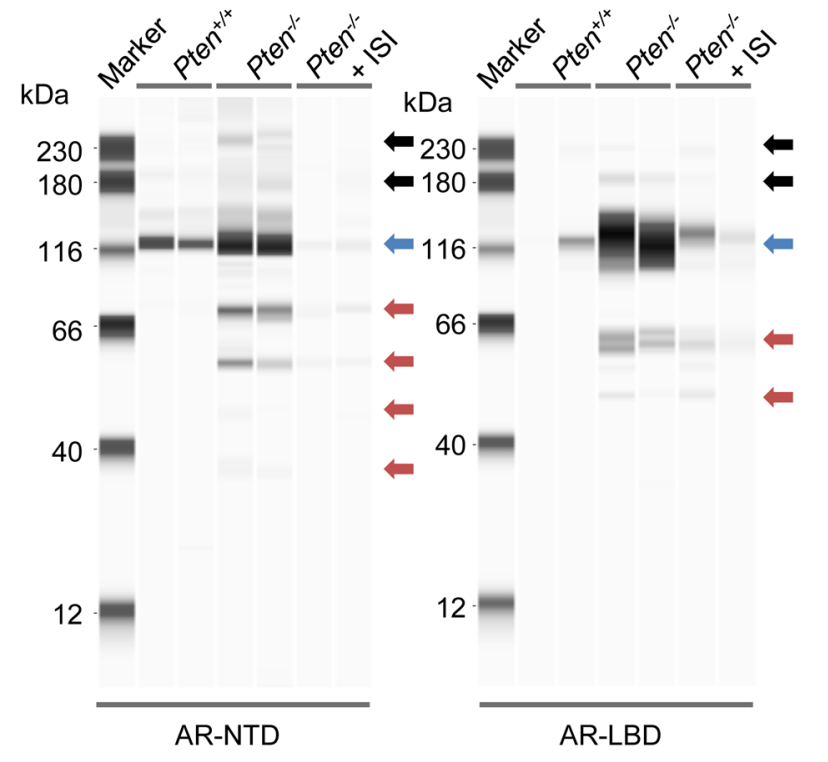

B

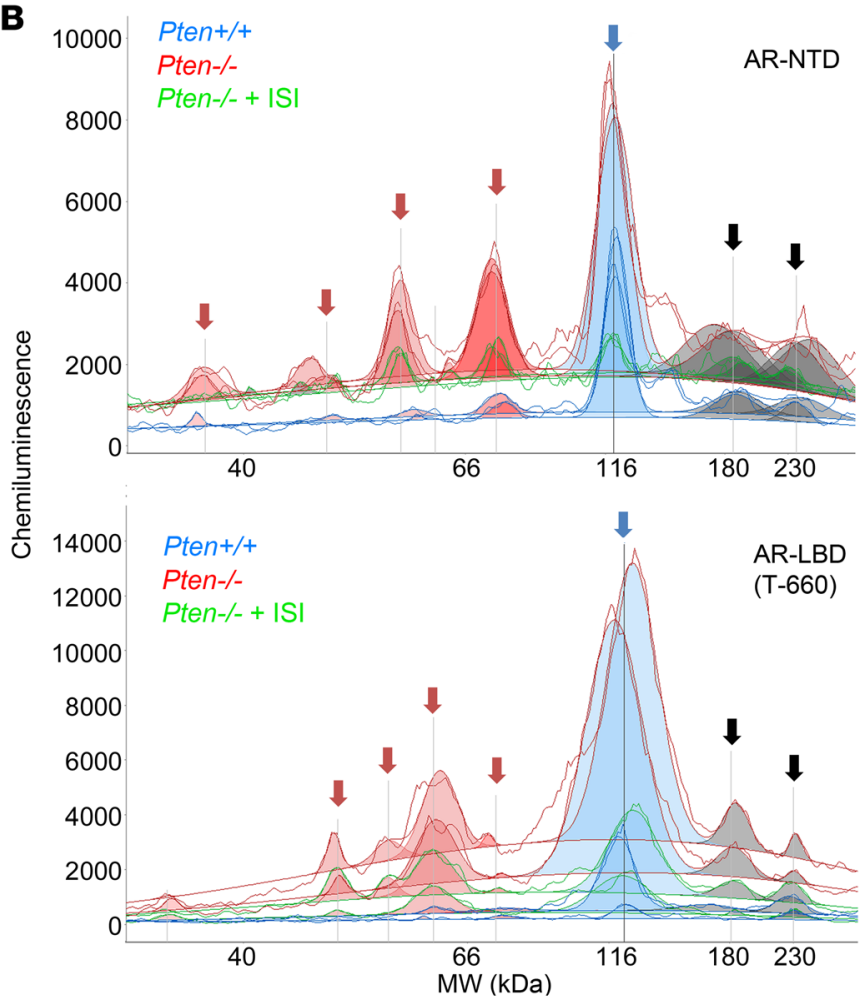

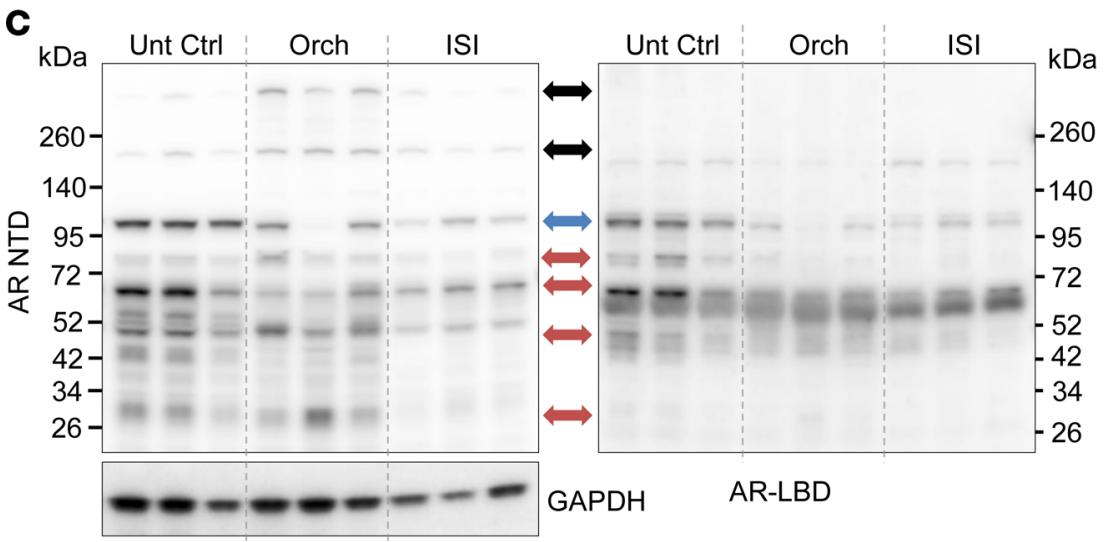

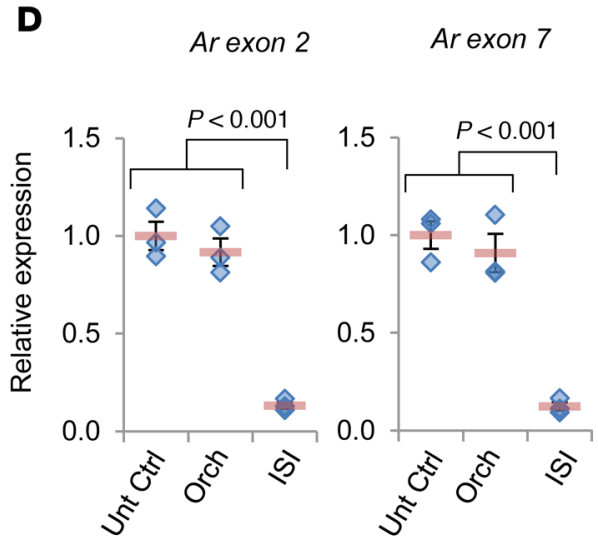

Figure 2. Generation-2.5 ASO targeting mouse Ar decreases expression of full-length AR and putative splice variants. Expression of full-length AR $(A R-F L)$ and aberrant $A R$ in mouse prostate tumors was determined by automated capillary electrophoresis with an $A R$ antibody targeting the $n$-terminal domain (AR-NTD, aa204-221) and the ligand binding domain (AR-LBD, aa660-899, see Supplemental Figure 3C) in prostates from 20-week-old normal WT $\left(\right.$ Pten $\left.^{+/+}\right)$mice or prostate tumors from untreated and ISIS581088-treated Pten-deficient $\left(\right.$Pten $\left.^{-/}\right)$mice. ISI581088 was administered for 4 weeks (40 $\mathrm{mg} / \mathrm{kg} / \mathrm{d}$ for first week loading, followed by 3 weeks of maintenance dosing, $40 \mathrm{mg} / \mathrm{kg} 3 \times /$ week). The results are shown as a virtual blot (A) and electropherogram (B). AR-FL, putative AR-Vs and high molecular weight bands are represented by blue, red, and black arrows, respectively. Shaded areas in the electropherogram denote peaks after baseline correction. (C) Western blot of AR expression using anti-AR-NTD and anti-AR-LBD antibodies in prostate tumors from untreated Pten-KO mice and 14 days after orchidectomy or 10 days of treatment with ISIS581088 (ISIS581088 was administered according to the dosing schedule in Supplemental Figure 1A). GAPDH was used a loading control. (D) Plots of Ar mRNA expression by qPCR. Horizontal bars represent mean \pm SEM, and diamonds represent individual samples; $n=3$ mice/group. Significance represent Student-Newman-Keuls post hoc test for individual comparisons, upon significant 1-way ANOVA ( $\left.\operatorname{ArEx2}, F_{2,8}=65.306, P<0.001 ; \operatorname{Ar} E x 7, F_{2,8}=46.706, P<0.001\right)$.

and chlormadinone acetate (CMA), a synthetic progestin with antiandrogenic and antigonadotropic effects $(2,3,33,34)$. For this experiment, mice were treated for 4 weeks, as in Figure 3A. Significant reductions of the GUT were observed for all treatments. With the exception of enzalutamide, atrophy of the GUT (which contains both tumor and normal tissue but is predominantly composed of normal tissue - namely seminal vesicles, coagulating gland, and bladder) was $\sim 40 \%$ for apalutamide-, abiraterone-, and CMA-treated mice compared with $48 \%$ for ISI581088-treated mice and almost 70\% for castrated mice (Figure 4, A-C). 
A

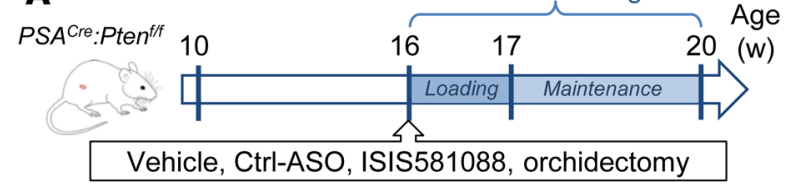

B

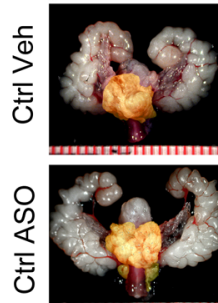

Ф :
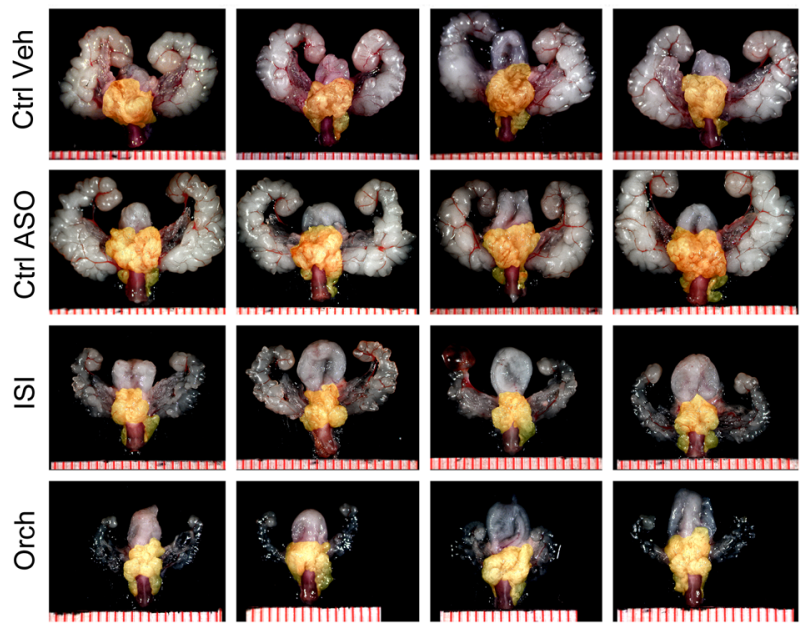

$\mathbf{F}$

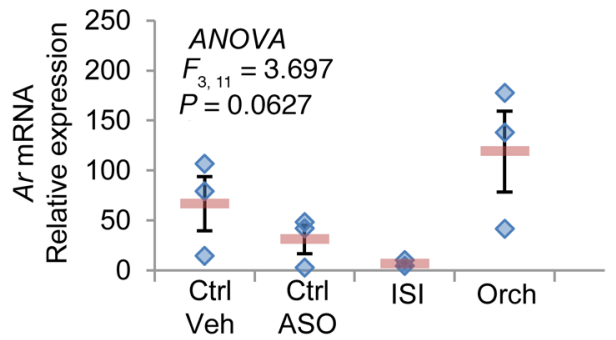

H
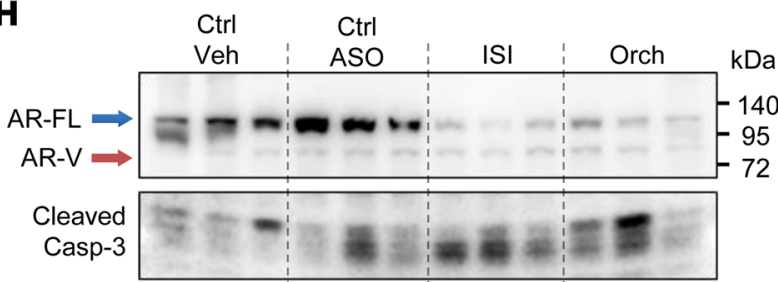

GAPDH

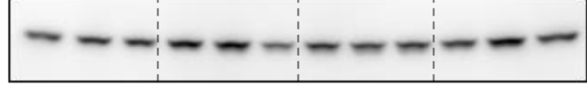

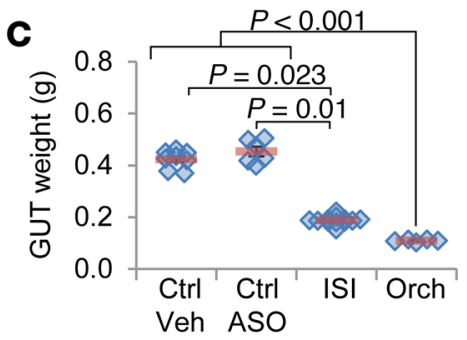

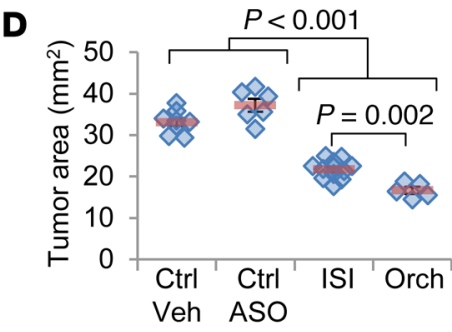

\section{E}
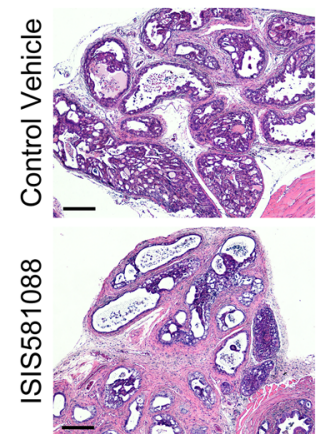

G
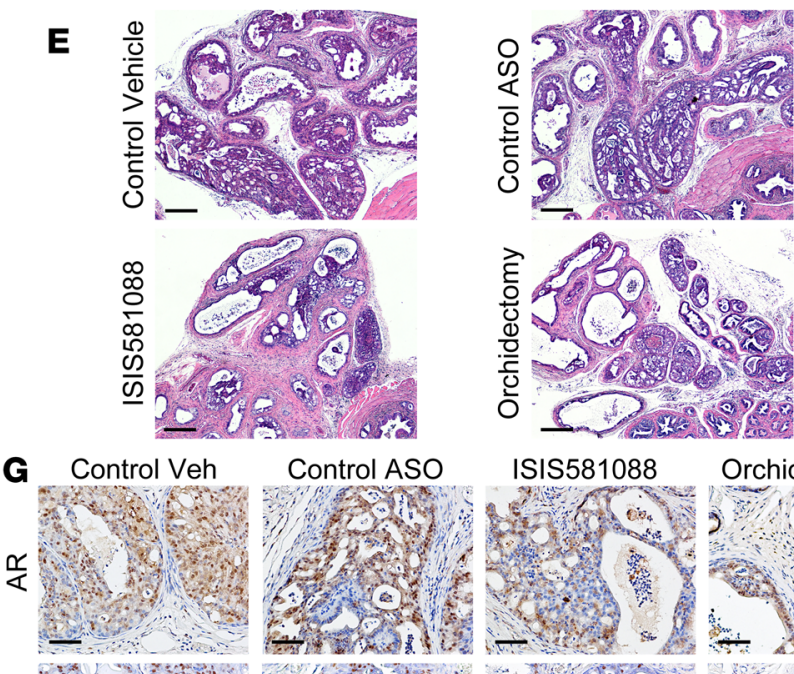

ISIS581088 Orchidectomy
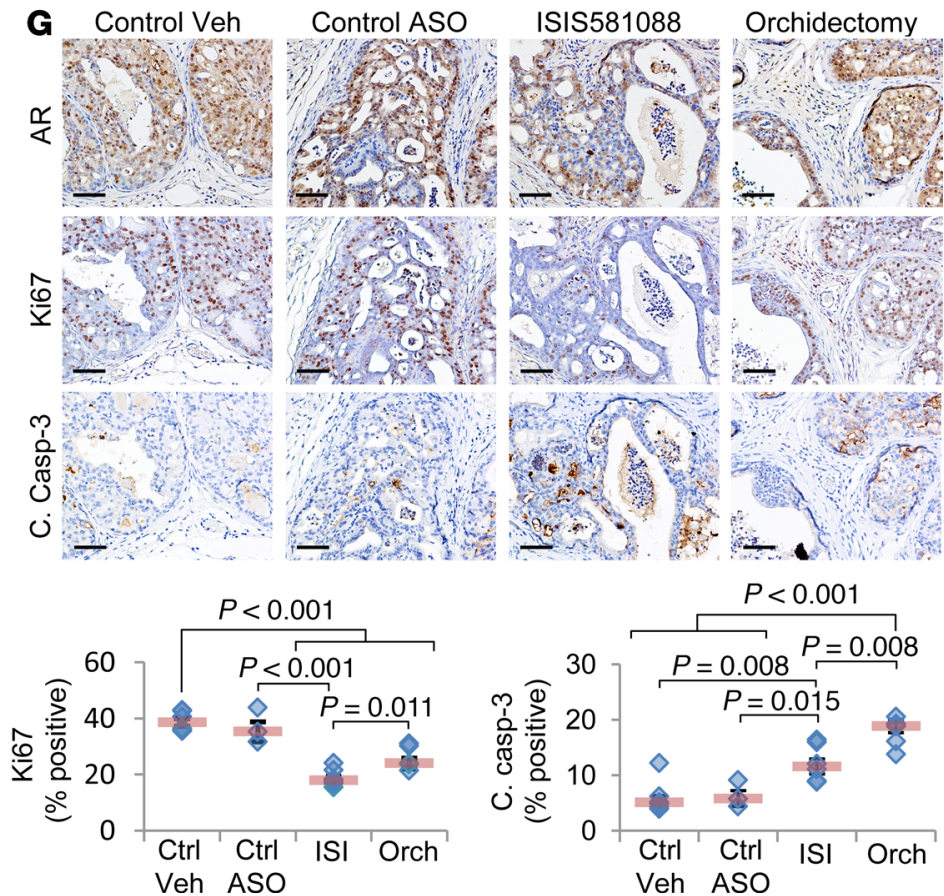

Figure 3. Antitumor activity of ISIS581088 in mouse Pten-deficient prostate tumors. (A) Experimental design for drug intervention model. Mice were randomized into vehicle-treated $(n=8)$, control ASO $(n=6)$, or ISIS581088 $(n=12)$ cohorts, and mice 4 weeks after surgical castration (Orch, $n=8)$ are also shown. Control ASO and ISIS581088 were administered i.p. for 4 weeks $(40 \mathrm{mg} / \mathrm{kg} / \mathrm{d}$ for first week loading, followed by 3 weeks of maintenance dosing, 40 $\mathrm{mg} / \mathrm{kg} 3 \times /$ week). Data represent the pooled analysis from 2 independent experiments using age-matched mice. (B) Representative images of genitourinary tracts (GUT). Prostate tumors are highlighted by a yellow mask; scale represents $\mathrm{mm}$. (C) Plots of GUT weight; horizontal bars represent mean \pm SEM, and diamonds represent individual samples. Significance represent Dunn's post hoc test for individual comparisons, upon significant 1-way ANOVA on ranks $\left(H_{3}=25.632, P<0.001\right)$. (D) Plots of prostate tumor area; horizontal bars represent mean $\pm \mathrm{SEM}$, and diamonds represent individual samples. Significance represent Student-Newman-Keuls post hoc test for individual comparisons, upon significant 1-way ANOVA $\left(F_{3,30}=82.965, P<0.001\right)$. (E) Representative H\&E-stained sections of mouse prostate tumors. Scale bars: $200 \mu \mathrm{m}$. (F) Plot of Ar mRNA expression determined by qPCR. Horizontal bars represent mean \pm SEM, and diamonds represent individual samples. (C) Representative IHC photomicrographs of AR, the proliferation marker Ki67, and apoptosis marker cleaved caspase- 3 in mouse prostate tumors. Plots represent percentage of positive-stained cells in epithelial cancer cells $(n=6$ mice/group); horizontal bars represent mean \pm SEM, and diamonds represent individual samples. Significance represent Student-Newman-Keuls post hoc test for individual comparisons, upon significant 1-way ANOVA (Ki67, $F_{3,19}=28.843, P<0.001$; c. casp-3, $F_{3,19}=16.276, P<0.001$ ). Scale bars: 100 $\mu$ m. (H) Confirmation of AR inhibition and apoptosis induction by Western blot expression of AR, cleaved caspase-3. GAPDH was used a loading control. 
These results indicate similar anti-AR activity in normal AR-responsive tissues between most of the pharmacological anti-AR interventions, including ISIS581088. Overall, ISIS581088 demonstrated the most potent antiandrogenic and antitumor activity of the pharmacological treatments. Consistent, with our previous results, tumor growth inhibition of ISIS581088 was comparable with that of orchidectomy; moreover, mice treated with ISIS581088 exhibited $52.2 \%, 36.5 \%, 36.8 \%$, and $48.0 \%$ reductions in prostate tumor weight compared with enzalutamide, apalutamide, abiraterone, and CMA, respectively (Figure 4B). Histological changes associated with androgen withdrawal were seen in all treatment groups, and the degree of change was commensurate with tumor growth inhibition (Figure 4C).

Next, we evaluated molecular responses to the various antiandrogen therapies on mouse prostate tumors using a focused qPCR-based approach analyzing a panel of 13 genes including $A r$ (targeting exons 2 and 7 and the exon 5-6 junction), core AR-responsive genes (Fkbp5, Nkx3.1, Msmb, Tmpress2, Timp4, Igfr1, and Klk4), AR-stimulating genes (Stat3, Myc, and Igf1), proliferation gene Mki67, and apoptosis genes (Casp3 and $B c l 2$ ). Thirteen of the 16 genes/transcripts were differentially expressed between the treatment groups (Supplemental Table 1). As expected, the mRNA levels of $A r$ were strongly inhibited by ISIS581088, which was also characterized by decreased expression levels of the AR-responsive genes Nkx3.1, Fkbp5, Tmprss2, Msmb, Timp4, and Igflr. Unsupervised hierarchical clustering of significant genes formed 3 distinct clusters (clusters A-C, Figure 4D). Clusters B and C were distinguished from cluster A, which includes 3 of the 4 untreated control mice. Cluster B showed the greatest reduction of $A r$-regulated mRNA expression and included all - ISIS581088, orchidectomized, and apalutamide-treated - mice, as well as 2 of 4 CMA-treated mice, 1 abiraterone-treated mouse, and 1 untreated control mouse. Conversely, cluster $\mathrm{C}$ demonstrated little to no downregulation of $A r$-regulated genes and was characterized with higher expression levels of $A r$. This cluster included all baseline and enzalutamide-treated mice, 3 of 4 abiraterone-treated mice, and 2 of 4 CMA-treated mice. Notably, mice in these treatment cohorts experienced the lowest antitumor response. It is well known that enzalutamide treatment leads to compensatory induction of AR/AR-Vs and its downstream targets (35). Figure 4D clearly shows that, suggesting that enzalutamide worked as predicted; therefore, lack of antitumor activity by enzalutamide is probably due to induction of AR. Overall, these results show that, in this context, Generation-2.5 Ar-ASO therapy is superior to other forms of pharmacological AR-targeting therapies.

Ar silencing exhibits antitumor activity in castration-resistant prostate tumors. Our next aim was to determine whether $A r$ silencing using Generation-2.5 Ar-ASO would inhibit mouse CRPC growth. In our Pten-deficient PCa mouse model, surgical castration fails to eradicate tumors and results in the development of mouse CRPC with persistent AR (25). For this analysis, we treated 16-week-old Pten-KO mice with castration-resistant prostate tumors (6 weeks after orchidectomy) with Ctrl-ASO or ISIS581088 for 4 weeks (Figure 5A). In this setting, normal accessory sex organs atrophied due to the androgen withdrawal, but tumors persisted (Figure 5B). Treatment with ISIS581088 did not result in additional tissue regression of already-atrophied normal accessory sex organs (Figure 5C). However, we did observe a small but statistically significant reduction of tumor burden in mice treated with ISIS581088 (Figure 5D). Histologically, mice treated with ISIS581088 contained fewer high-grade mPIN lesions (Figure 5E). No significant changes in tumor cell proliferation rates were seen after treatment with the ISIS581088 (Figure 5F); however, a significant increase in the percentage of cleaved caspase-3-positive cells in these mice confirmed the degeneration of epithelial cells (Figure 5, G and J). In this setting, treatment with ISIS581088 still led to strong silencing of $A r$ mRNA and reduced AR protein expression (Figure 5, H-J). Additionally, mice treated with ISIS581088 showed reduced expression of both AR-FL and the $\sim 80 \mathrm{kDa}$ AR-V and were associated with cleaved caspase-3 upregulation (Figure 5I). These results indicate that mouse castration-resistant prostate tumors still rely, at least in part, on AR for survival, and this dependence can be exploited with treatment using Generation-2.5 ASO targeting $A r$.

Ar silencing induces distinct transcriptional programs in murine Pten-deficient intact and castration-resistant prostate tumors. AR plays distinct roles in normal prostate development and function, and - during tumorigenesis and disease progression - AR modulates distinct transcriptional programs (35-37). Recent studies show that AR can dynamically regulate gene transcription by epigenetic regulation of DNA methylation of specific target genes (38). Thus, we aimed to investigate the effects of $A r$ silencing on AR transcription programs in castration-naive prostate tumors and CRPC. For this, we performed gene expression analysis against a panel of 86 mouse $A r$-responsive genes in mouse CNPC and CRPC treated with ISIS581088. Overall, ISIS581088 appeared to be more active in CNPC than CRPC and strongly downregulated 39 of the 86 AR-dependent genes, including the core target genes Nkx3.1, Fkbp5, Tmprss2, and Timp4 (Figure 6A and Supplemental Table 2). Consistent with our previous results, treatment with ISIS581088 significantly reduced the levels of $A r$ mRNA in both 

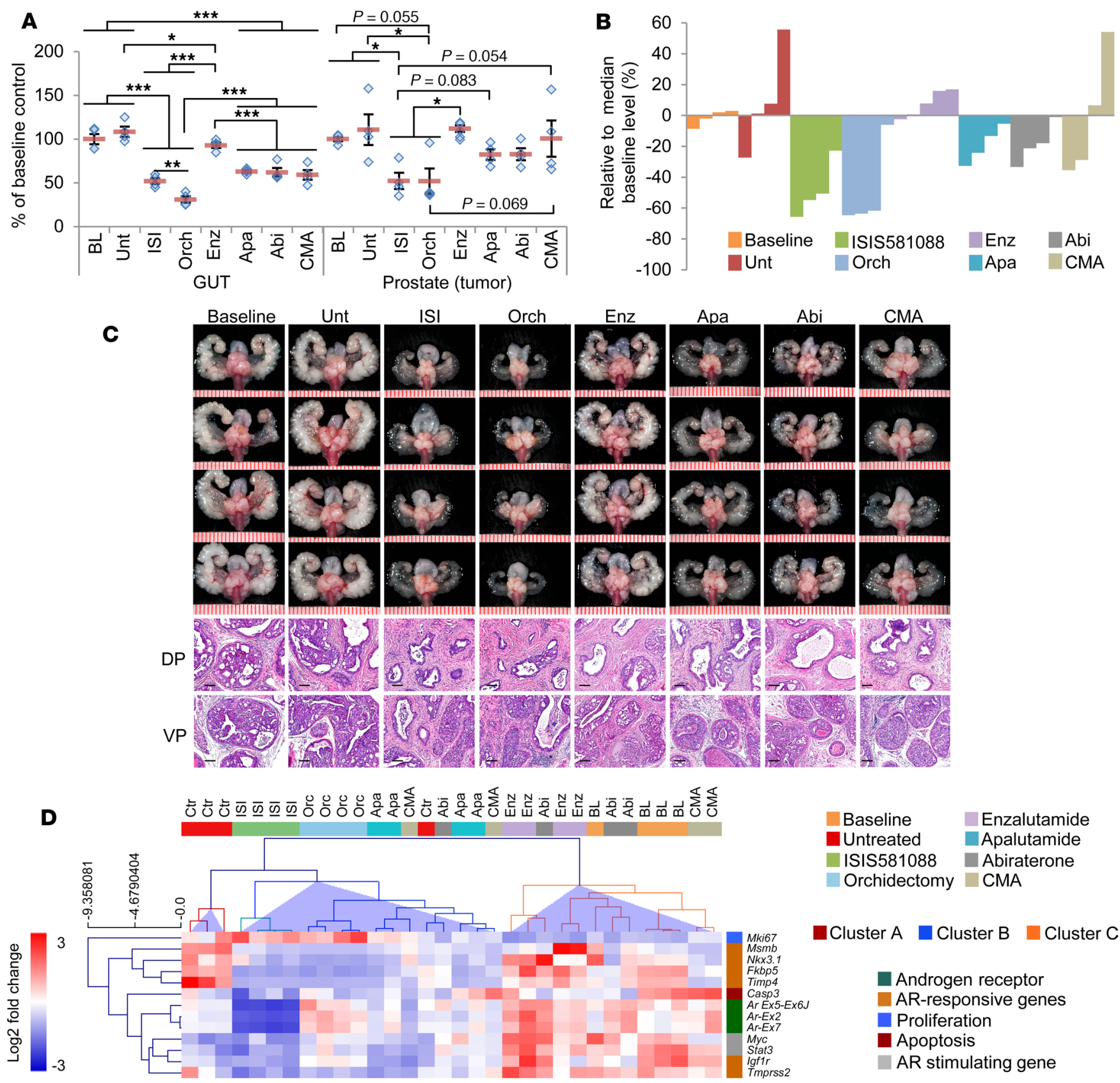

Cluster A $\square$ Cluster B $\square$ Cluster C

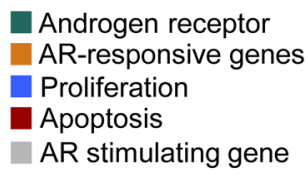

Figure 4. Ar-ASO blockade is more effective at suppressing mouse Pten-deficient prostate tumor growth compared with other pharmacological antiandrogen therapies. The activity of ISIS581088 was compared with surgical castration and pharmacological AR inhibition. Pten-KO mice (32 weeks of age) were randomized as control or were assigned to ISIS581088 (ISI, 40 mg/kg, 1 week loading, 3 weeks maintenance), orchidectomy (Orch), enzalutamide (Enz, 30 mg/kg/ day), apalutamide (Apa, $30 \mathrm{mg} / \mathrm{kg} /$ day), abiraterone (Abi, $200 \mathrm{mg} / \mathrm{kg} /$ day), and chlormadinone acetate (CMA, $100 \mathrm{mg} / \mathrm{kg} /$ day) treatment groups. Mice received treatment for 4 weeks, and untreated littermates were collected at baseline (BL), $n=4$ mice/group. (A) Plots of mean \pm SEM of GUT and prostate weights normalized to baseline means. Horizontal bars represent mean \pm SEM, and diamonds represent individual mice. Significance represent Student-Newman-Keuls post hoc test for individual comparisons, upon significant 1-way ANOVA (GUT, $F_{7,32}=36.25, P<0.001 ;$ prostate, $F_{7,32}=4.162, P<0.001$ ); ${ }^{*} P<0.05 ;{ }^{* *} P<0.01$; ${ }^{* * *} P$ $<0.001$. (B) Waterfall plot of individual treatment response based on prostate tumor weight normalized to median baseline level. (C) Representative images of whole genitourinary tracts and $\mathrm{H} \& \mathrm{E}$-stained sections of mouse dorsal prostate (DP) and ventral prostate (VP) tumors. Scale in GUT represents mm (scale bars: $100 \mu \mathrm{m})$. Scale in GUT represents mm (scale bars: $100 \mu \mathrm{m}$ ). (D) Clustering analysis of differentiated expresses genes from qPCR-based panel Ar, core AR responsive, AR stimulating, proliferation, and apoptosis genes. Heatmap shows unsupervised hierarchical clustering using complete linkage and Pearson correlation.

CRPC and CNPC. Genes significantly downregulated in CNPC are largely involved in cellular metabolic processes, including cholesterol metabolism, steroid biosynthesis, and response to hormone and insulin stimulus (Figure 6B). In CRPC, only 4 of the 86 genes downregulated reached statistical significance: Ar, Mme, Mki67, and $M f d 2$. Of these, $M k i 67$ encodes the proliferation marker Ki67, and Mme encodes the cell surface marker 


\section{A}

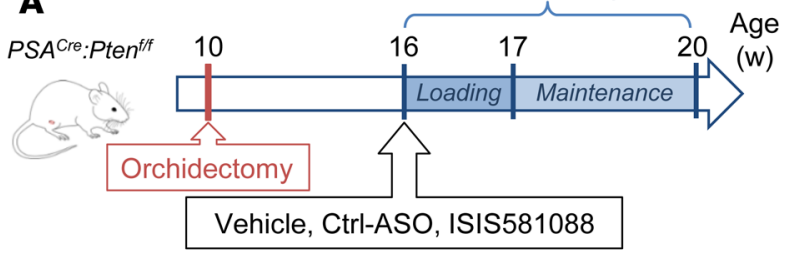

B
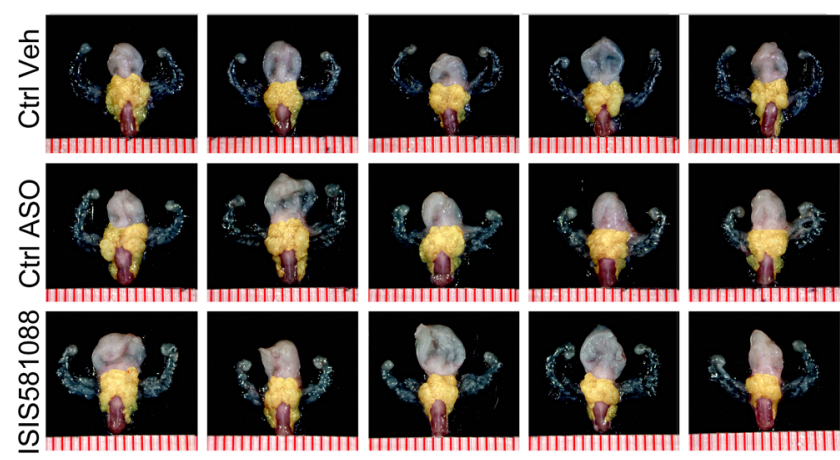

C
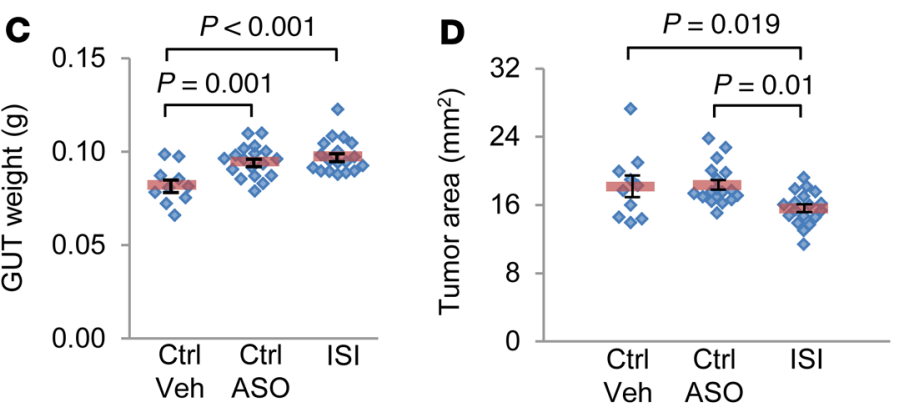

E

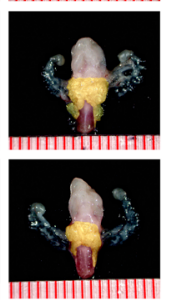

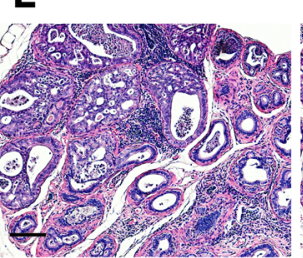

Control Veh

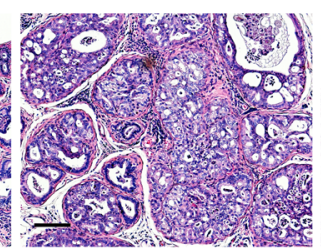

Control ASO

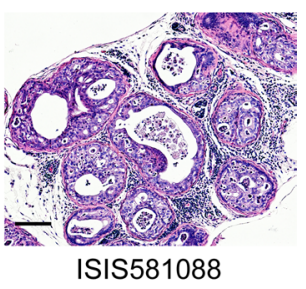

H

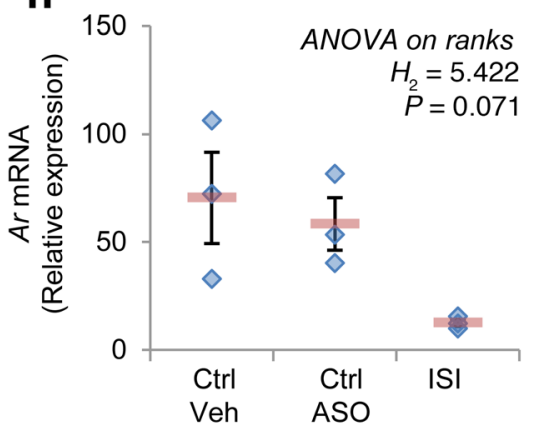

I
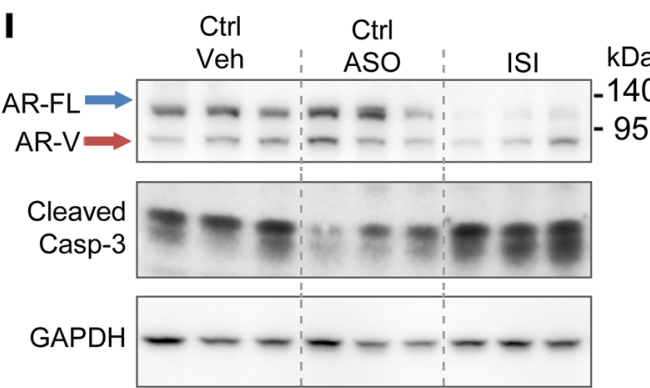

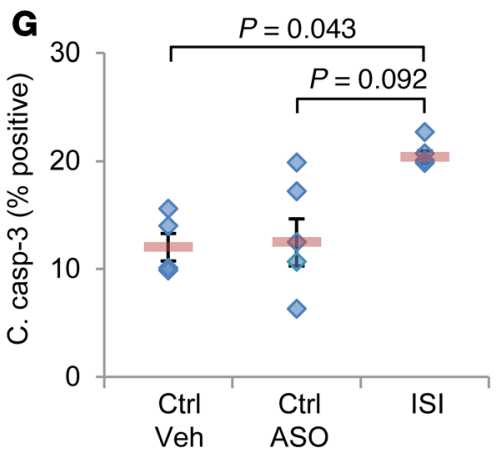

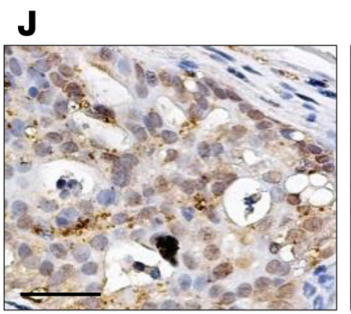

Control Veh

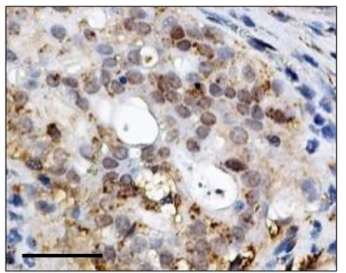

Control ASO

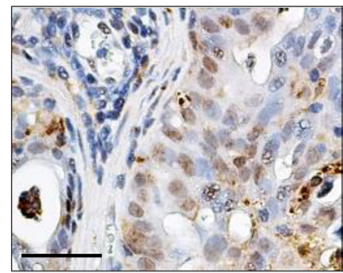

ISIS581088

Figure 5. ISIS581088 demonstrates antitumor activity against murine Pten-deficient castration-resistant prostate tumors. (A) Experimental design for drug intervention studies using castration-resistant prostate tumors in conditional Pten-KO mice. Mice were randomized to vehicle $(n=$ 10), control ASO $(n=18)$, or ISIS581088 $(n=18)$ treatment groups 6 weeks after surgical castration. Control ASO and ISIS581088 were administered i.p. for 4 weeks ( $40 \mathrm{mg} / \mathrm{kg} / \mathrm{d}$ for first week loading, followed by 3 weeks of maintenance dosing, $40 \mathrm{mg} / \mathrm{kg} 3 \times /$ week). Data represent the pooled analysis from 2 independent experiments using age-matched mice. (B) Representative images of genitourinary tracts (GUT). Prostate tumors are highlighted by a yellow mask; scale represents $\mathrm{mm}$. Plots of GUT weight (C) and tumor area (D). Horizontal bars represent mean \pm SEM, and diamonds represent individual mice; significance represent Student-Newman-Keuls post hoc test for individual comparisons, upon significant 1-way ANOVA. $F_{2,45}=9.463, P<0.001$ (C); $F_{3,45}=5.538, P=0.007$ (D). (E) Representative H\&E-stained sections of mouse prostate tumors. Scale bars: 200 $\mu \mathrm{m}$. Plots of proliferation (Ki67) (F) and apoptosis (cleaved caspase-3) (G) in prostate epithelial cancer cells; $n=4-6$ mice $/$ group. Horizontal bars represent mean $\pm S E M$, and diamonds represent individual samples. Significance in $\mathbf{G}$ represent Dunn's post hoc test for individual comparisons, upon significant 1-way ANOVA on ranks $\left(H_{2}=7.058, P=0.018\right)$. (H) Plot of $A r$ mRNA expression determined by qPCR. Horizontal bars represent mean \pm SEM, and diamonds represent individual samples. (I) AR downregulation and induction of apoptosis were examined by Western blot. GAPDH was used a loading control. (J) AR downregulation was confirmed by immunohistochemical staining of AR. Scale bars: $100 \mu \mathrm{m}$. 
cluster of differentiation 10 (CD10), which has been shown to be associated with cancer progression and poor clinical outcomes in various cancers, including prostate $(39,40)$. Since many of the AR target genes share multiple transcriptional regulators, a lack of responsiveness to ISI581088 in the CRPC setting could be due to redundant transcriptional pathways activated in the absence of androgens. We also observed some activity with the Ctrl-ASO in a CNPC setting, and to a lesser degree in CRPC, although none were statistically significant.

In order to gain more insight into the effects of $A r$-silencing in castration-naive and -resistant prostate tumors, we performed gene interaction and enrichment analysis using genes that were downregulated by less than or equal to $-0.5 \log _{2}$ fold-change vs. control mice. A total of $51(59 \%)$ and $29(34 \%)$ genes were downregulated in CNPC and CRPC, respectively, and of these, only 17 (27\%) genes were common between CNPC and CRPC (Figure 7A). Functional enrichment analysis of downregulated genes demonstrated distinct signatures between the CNPC and CRPC phenotypes (Figure 7B and Supplemental Table 3). These results provide evidence for distinct in vivo AR transcriptional programs between intact and castration-resistant Pten-deficient prostate tumors and provide insight into the complexity of AR-responsive regulatory networks.

Antitumor efficacy of Generation-2.5 Ar-ASO in combination with AKT inhibition in Pten-deficient prostate tumors. Given the evidence of reciprocal feedback regulation between AR and PI3k/AKT signaling, we next aimed to determine the efficacy of combined AR and PI3/AKT pathway blockade using the Generation-2.5 Ar-ASO, in combination with the pan-AKT inhibitor AZD5363, in our Pten-deficient PCa model. In vitro studies using mouse PCa cell lines showed synergistic activity with this treatment combination (Supplemental Figure 5). In the mouse CNPC intervention model, combination therapy with ISIS581088 and AZD5363 significantly reduced tumor burden compared with monotherapy (Figure 8A and Supplemental Figure 6A). Whereas monotherapy with AZD5363 appeared to yield only a cytostatic effect on tumor growth, treatment with ISIS581088 produced slight tumor regression, and this was enhanced with combination therapy (Supplemental Figure 6C). Further analysis showed that combination therapy did, indeed, enhance the tumor antiproliferative and apoptotic effects over monotherapy (Figure 8, B and C). In the CRPC intervention model, combination therapy did not demonstrate improved antitumor activity over monotherapy (Figure 8D and Supplemental Figure 6, B and D). Similarly, we did not observe an improvement in the suppression of tumor proliferation after combination therapy (Figure 8E). However, we did see a significant enhancement in the induction of apoptosis with combination therapy (Figure $8 \mathrm{~F}$ ). Histologically, tumors from CNPC mice receiving combination therapy exhibited a higher degree of atrophied mPIN glands and fewer large branched mPIN glands compared with mice treated with monotherapy (Figure $8 \mathrm{G}$ ). While we did not observe gross changes in tumor burden between mice treated with combination therapy and monotherapy in the CRPC model, we did, indeed, note some histological changes. Overall, responses varied among mice, but we did observe remarkable changes in some mice, such as an increase in the number of cystic glands replete with apoptotic cells in the lumens and almost complete atrophy in 1 animal (Figure 8H).

Further characterization of molecular responses to ISIS581088 and AZD533 therapy in prostate tumors were examined by Western blot and qPCR analysis. As expected, monotherapy with ISIS581088 alone or in combination with AZD5363 effectively inhibited AR mRNA and protein levels in both CNPC- and CRPC-treated mice (Figure 9, A-D). Western blot analysis revealed increased AR protein levels after treatment with AZD5363 in both CNPC and CRPC models, and treatment with ISIS581088 increased in the phosphorylation of AKT in both CNPC and CRPC models; however, the phosphorylation of the AKT pathway downstream proteins, PRAS40 and S6 proteins, was greater in CRPC mice (Figure 9, A and B). AR transcriptional activity was determined by gene expression analysis of 6 core mouse AR-responsive genes (Nkx3.1, Fkbp5, Tmprss2, Timp4, Klk4, and Msmb). Overall, inhibition of AKT signaling tended to lessen the suppression of AR-responsive gene mRNAs modulated by ISIS581088 in castration-naive prostate tumors (Figure 9E). However, in CRPC, suppression of the mRNA levels of AR-responsive genes by ISIS581088 tended to be better when AZD5363 was coadministered (Supplemental Figure 7). These findings provide additional evidence for reciprocal feedback regulation in prostate tumors and show that this manifestation may be more prominent in the CRPC setting. More importantly, we show that AR and PI3/AKT pathway blockade with $A r$-ASO and AKT kinase inhibition has the potential to improve therapeutic response.

Combination therapy of ISIS581088 and AZD5363 overcomes monotherapy resistance in mouse advanced PCa. Although we did not observe a reduction of tumor burden with the combination of ISIS581088 and AZD5363 in our early-stage Pten-deficient CRPC model, the combination therapy did, indeed, demonstrate the ability to inhibit downstream signaling to enhance apoptosis. Therefore, we could not 
A
$\bar{m}$
$\stackrel{N}{N}$
ָ̃
ì
Linear fold chanae

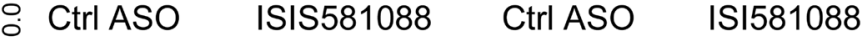

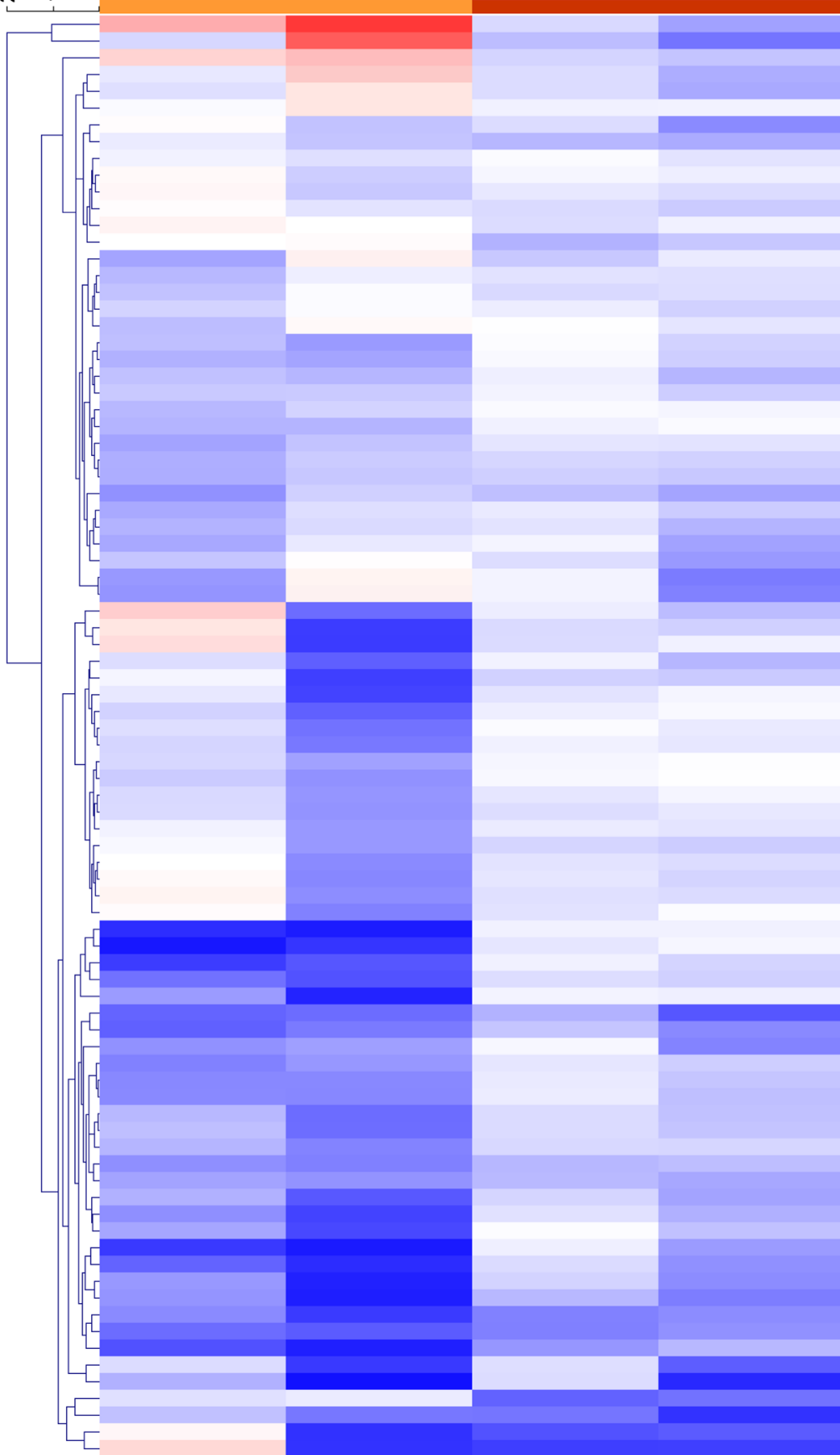

$T p d 52$ Cxcl1 Psph Insig2 Cyp2f 2 Map 2k4 Myc

Wwtr1
Psmd1 Psmd Erp44

Abcc4

Ldha

Pkd211

Griqc

Tax1bp1

Slc40a1

Tmprss2

Nelf

Herc3
Limk2
Fas

Parp1

Tmem45

Sgk3

Ndgrt

Pparge1

Zfpargc1a

Alox12

Alox12

Twist

Cxcl1

Sgk1

Doc2b

Pdlim2

Fkbp5

Halpn

lqgap2
Dhcr24

Dhcr24
Pdia4

Pdia4
Dher 7

Hif1a

Fkbp

Cre

Dnajc3

Sqle

Epn2

Greb1

Gsdma1

Pkm2

Rasgrf

Mthrd2

SIc2a 4

Ubec 2

Ank1

Hmgsc1

Hmgsc1

Stk 39

Pfkfo

B4gals

Mki67

Der/3

Gnmt

Mfsd2

Sic38a5

$\mathrm{Scd} 4$

Aldh112

Ar

Camk 2

Crabp1

$A b 0$

B

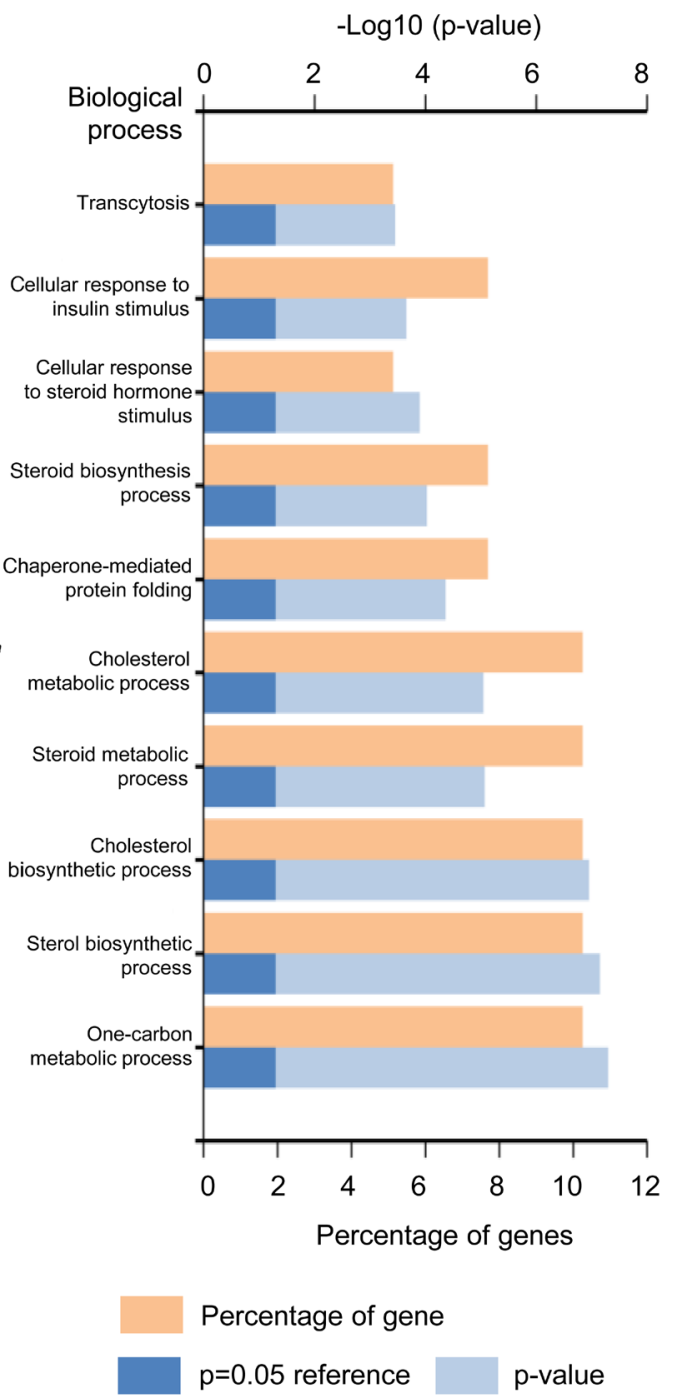

CNPC

CRPC

Figure 6. Expression profiling of AR-responsive genes in mouse Pten-deficient prostate tumors treated with ISIS581088. Sixteen-week-old mice with castration-naive (CNPC) or castration-resistant prostate tumors (CRPC, 6 weeks after surgical castration) were treated with saline $(n=5)$, control ASO $(n=6)$, or ISIS581088 $(n=6)$ for 4 weeks as described in Figure 3A and Figure 5A. Prostate tumors were collected for multiplex gene expression analysis of AR responsive genes using the Fluidigm analysis platform. (A) Heatmap and unsupervised hierarchical clustering (average linkage, Euclidean distance metric) of 86 AR-responsive genes after Ar silencing with ISIS581088 (see Supplemental Table 2). (B) Top biological processes enriched after Ar silencing in castration-naive prostate tumors. 
A

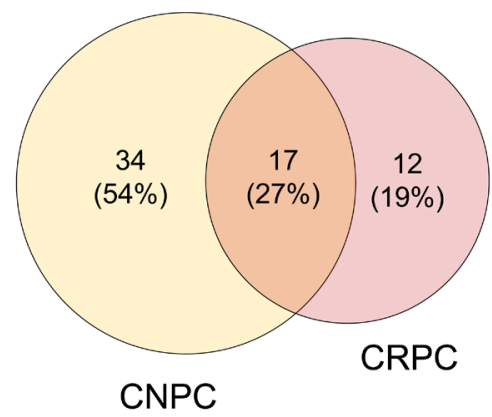

Downregulated in CNPC

\begin{tabular}{|cccc|}
\hline Creld2 & Fkbp11 & Mat2a & Rasgrf1 \\
Dbi & Fkbp5 & Mthfd2 & Sgk1 \\
Dhcr24 & Greb1 & Nelf & Sqle \\
Dhcr7 & Gsdma & Nkx3-1 & Stk39 \\
Dnajc3 & Hapln4 & Pdia4 & Timp4 \\
Doc2b & Hif1a & Pdk4 & Tmem97 \\
Epas1 & Hmgcr & Pdlim2 & Tmprss2 \\
Epn2 & Hmgcs1 & Pfkfb4 & Uso1 \\
& lqgap2 & Pkm2 & \\
\hline
\end{tabular}

Downregulated in CNPC and CRPC

\begin{tabular}{|cc|} 
Abo & Mfsd2 \\
Actg2 & Mki67 \\
Aldh1/2 & Mme \\
Ank1 & Phf19 \\
Ar & Scd4 \\
Crabp1 & S/c2a4 \\
Derl3 & Slc38a5 \\
Gnmt & Ube2c \\
Grb10 & \\
\hline
\end{tabular}

Downregulated in CNPC and CRPC

\begin{tabular}{|cc}
\hline Alox12 & Myc \\
Camkk2 & Ndrg1 \\
Cxcl11 & Pgam1 \\
Cxcl17 & Tpd52 \\
CYP2F1 & Twist1 \\
Insig2 & Wwtr1 \\
& \\
\hline
\end{tabular}

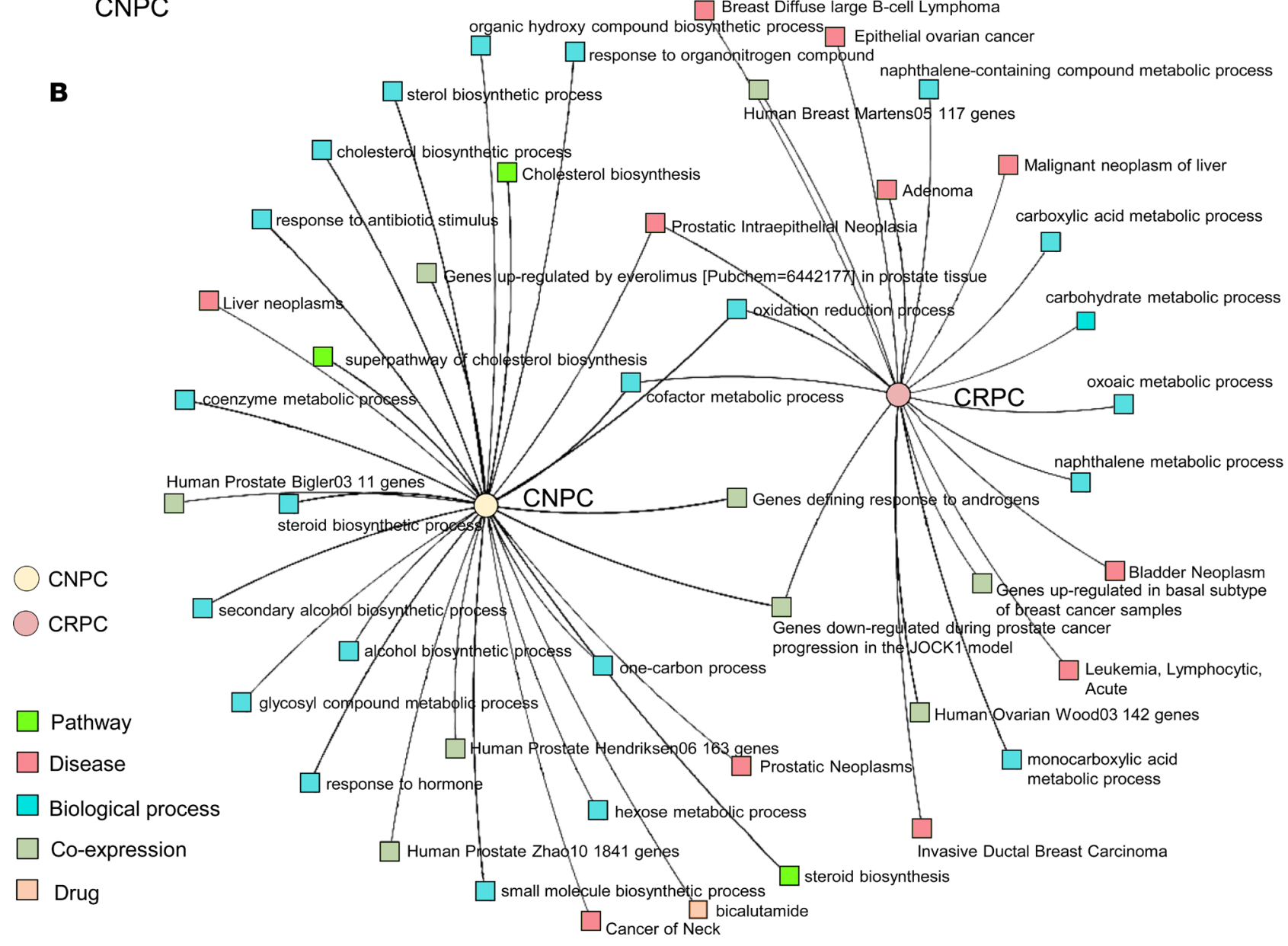

Figure 7. In vivo Ar silencing evokes differential AR transcriptional programs in mouse Pten-deficient prostate tumors. The gene set from Figure 6 was used to compare transcriptional programs between castration naive (CNPC) and castration-resistant prostate cancer (CRPC) phenotypes. (A) Venn diagram and gene list of genes downregulated (less than - 0.5 -fold) in mouse CNPC and CRPC after Ar gene silencing with ISIS581088. (B) Multicluster gene enrichment networks (generated by ToppCluster; see Supplemental Table 4) of mouse phenotypes after treatment with ISIS581088.

abandon the possibility that a therapeutic benefit could be achieved. To test this notion, we examined therapeutic response of ISIS581088/AZD5363 combination therapy in a clinically relevant model of advance CRPC that utilized clinically appropriate endpoints, such as overall survival (OS) (26). For

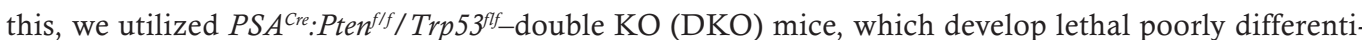
ated prostate tumors, in a mouse clinical trial model. We first compared the effects of $A r$-ASO silencing on OS with those of orchidectomy on DKO mice with advanced PCa. In this treatment model, mice were randomized to treatment when primary tumors became palpable and were monitored to term (Figure 10A and Supplemental Table 3). Although both Ar silencing using ISIS581088 and surgical castration showed activity in mice with advanced prostate tumors, neither treatment was sufficient to significantly improve OS or progression-free survival (PFS) (Figure 10B and Supplemental Figure 8A). 
A

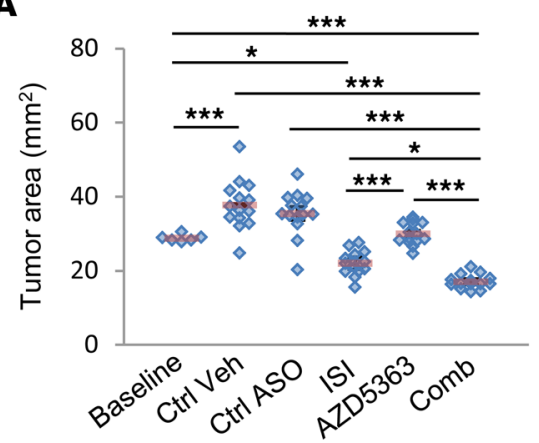

D

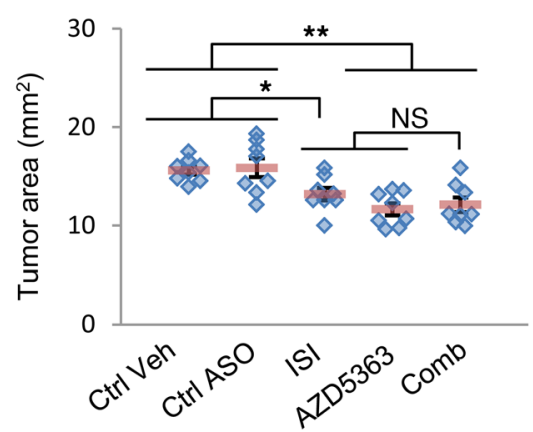

B

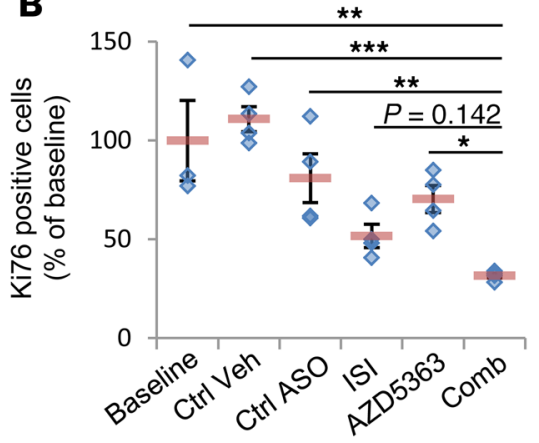

E

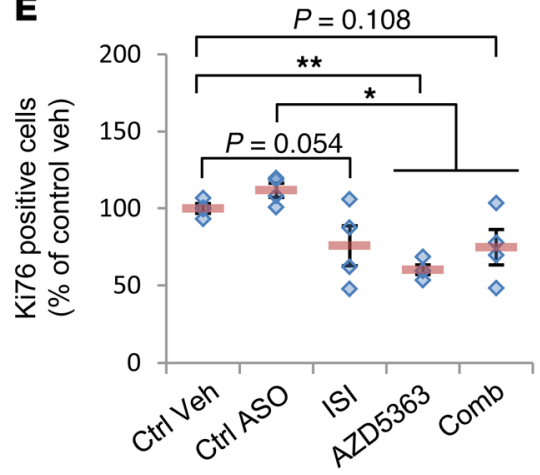

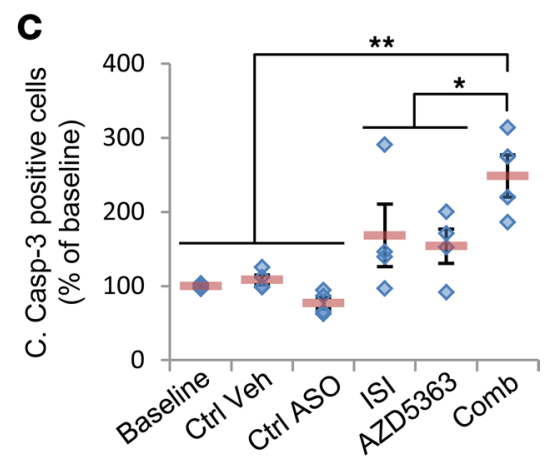

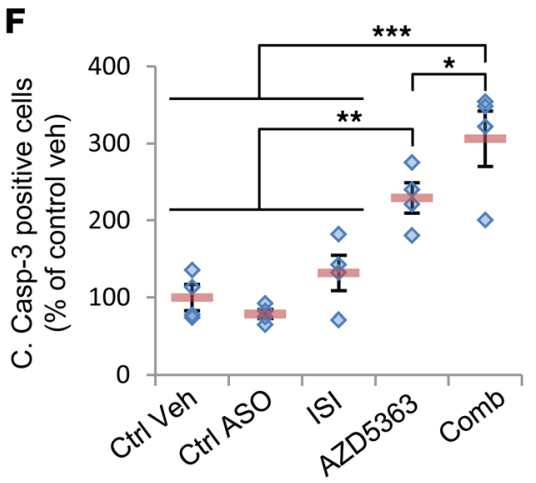

G

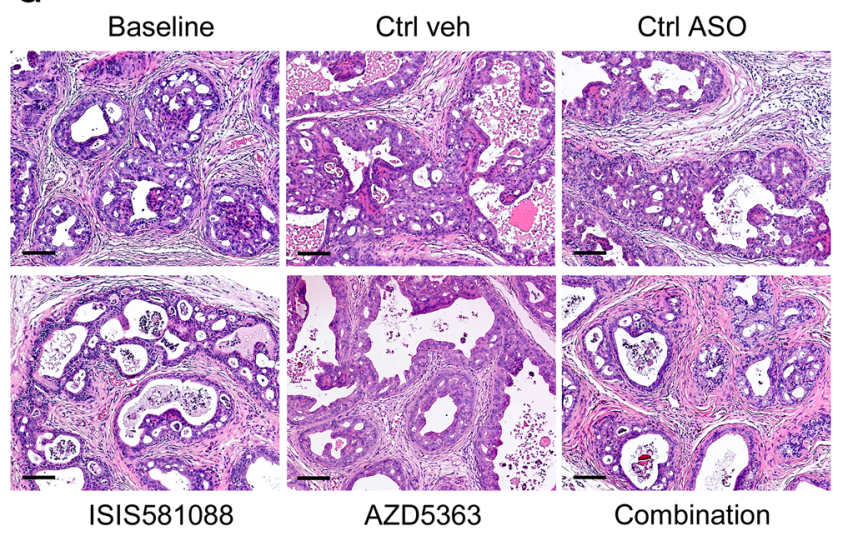

H

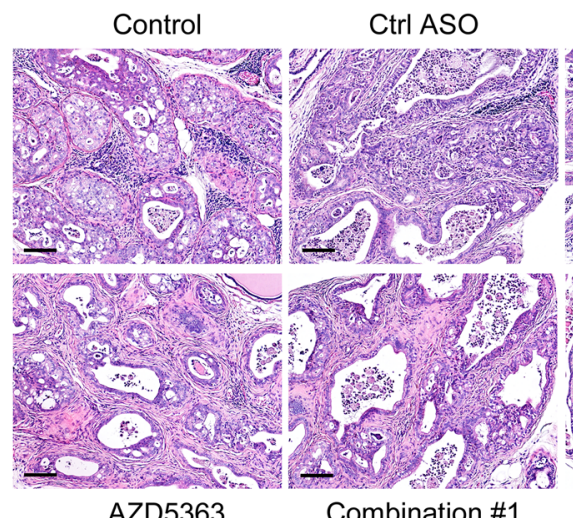

AZD5363

Combination \#1
ISIS581088

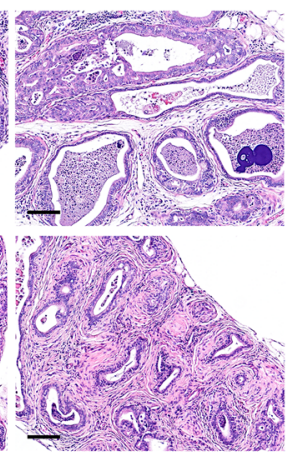

Combination \#2

Figure 8. Efficacy determination of in vivo combination therapy with ISIS581088 and AZD5363 in mouse Pten-deficient prostate cancer models. (A) Sixteen-week-old conditional Pten-KO mice were randomized into baseline $(n=6)$ and KLEPTOSE/DMSO as vehicle control (Ctrl veh), control ASO (Ctrl-ASO, $40 \mathrm{mg} / \mathrm{kg}$ ), ISIS581088 (ISI, $40 \mathrm{mg} / \mathrm{kg}$, see Figure 3A for ASO dosing schedules), AZD5363 (100 mg/kg b.i.d., p.o.), and ISIS581088+AZD5363 treatment groups as previously described. Plot represents pooled analysis of tumor burden from 2 independent studies; $n=14$ mice/group. Horizontal bars represent mean \pm SEM, and diamonds represent individual mice. Significance represent Student-Newman-Keuls post hoc test for individual comparisons, upon significant 1-way ANOVA $\left(F_{5,75}=44.277, P<0.001\right)$. Plots of tumor epithelial cell proliferation (B) and apoptosis (C) determined by Ki67 and cleaved caspase-3 IHC analyses; $n=3-4$ mice/group. Horizontal bars represent mean \pm SEM, and diamonds represent individual samples. Significance represent Student-Newman-Keuls post hoc test for individual comparisons, upon significant 1-way ANOVA (Ki67, $F_{5,22}=9.909, P<0.001$; c. casp-3, $F_{5,22}=6.627, P=0.001$ ). (D) Plot of tumor burden in 16-week-old mice with Pten-deficient castration-resistant prostate tumors (see Figure $5 \mathrm{~A}$ ) were randomized into vehicle control and indicated treatment groups as described above. Horizontal bars represent mean \pm SEM, and diamonds represent individual mice. Significance represent Student-Newman-Keuls post hoc test for individual comparisons, upon significant 1-way ANOVA ( $F_{4,39}$ $=8.177, P<0.001)$. Plots of tumor epithelial cell proliferation $(\mathbf{E})$ and apoptosis $(\mathbf{F})$ determined by Ki67 and cleaved caspase-3 IHC analyses. Horizontal bars represent mean \pm SEM, and diamonds represent individual samples; $n=4$ mice/group. Significance represent Student-Newman-Keuls post hoc test for individual comparisons, upon significant 1-way ANOVA (Ki67, $F_{4,19}=6.505, P=0.003$; c. casp-3, $F_{4,19}=18.672, P<0.001$ ). Representative $\mathrm{H} \& \mathrm{E}$-stained sections of mouse prostate tumors are shown with the indicated treatment for the castration-naive $(\mathbf{H})$ and castration-resistant prostate cancer models (G). Scale bars: $100 \mu \mathrm{m}$; ${ }^{*} P<0.05$; ${ }^{* *} P<0.01 ;{ }^{* *} P<0.001$. 
A

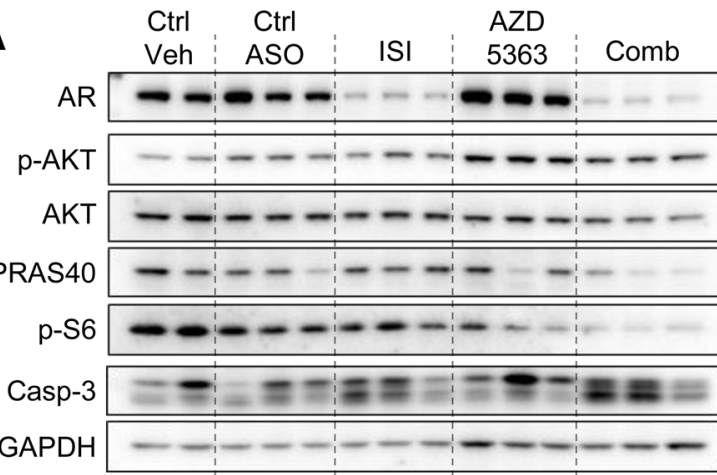

C

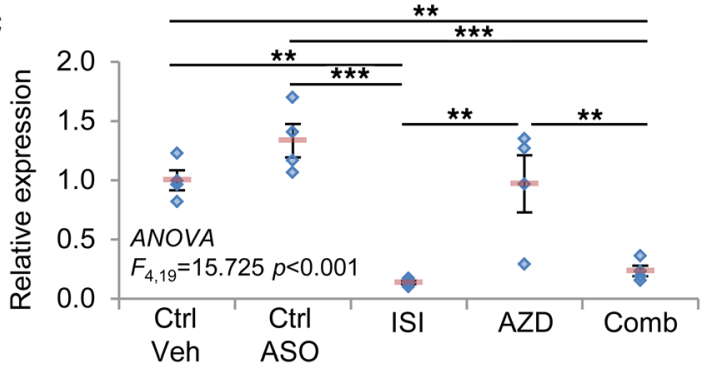

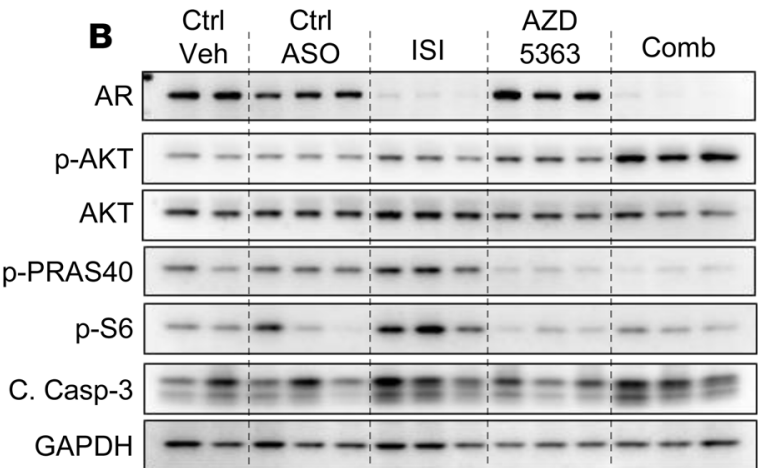

D

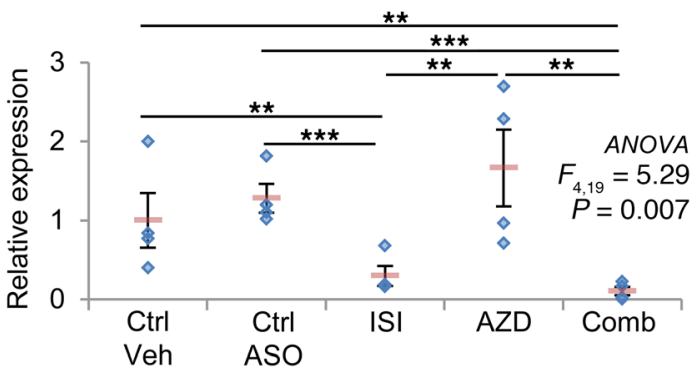

E
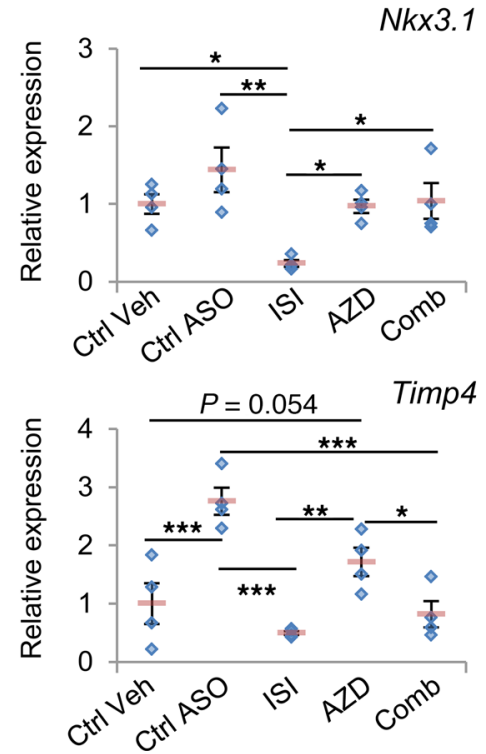

$N k \times 3.1$

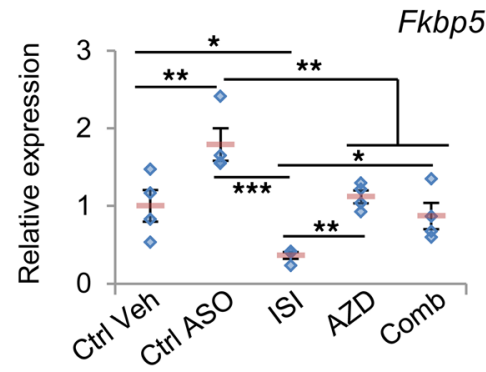

KIk4

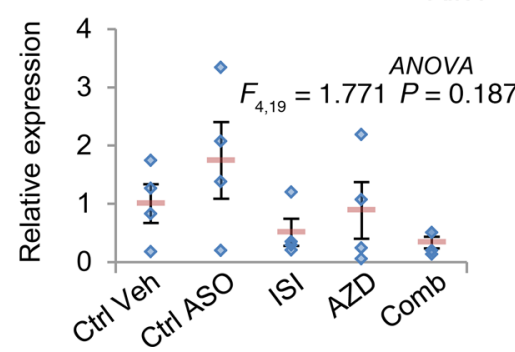

Tmprss2
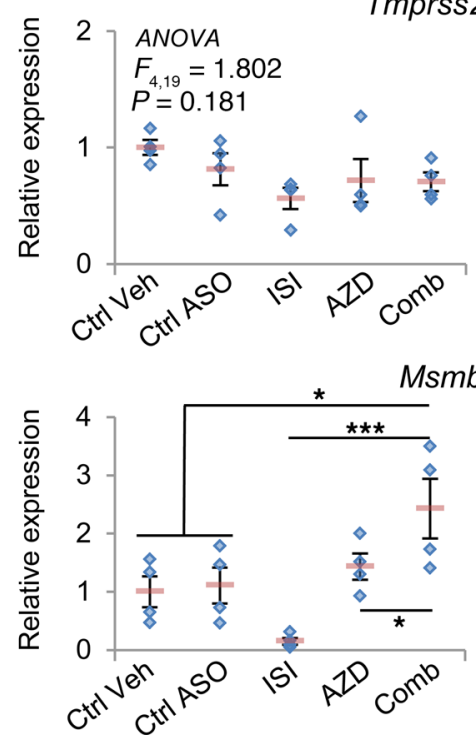

Figure 9. Molecular characterization of in vivo combination therapy with ISIS581088 and AZD5363 in mouse Pten-deficient prostate cancer models. Western blot analysis of AR, AKT, phosphorylation of AKT downstream molecules, and cleaved caspase-3 was performed in prostate tumors from representative mice in Figure 8. Prostates from mice in castration-naive (A) and castration-resistant (B) prostate cancer intervention trial were collected 4 hours after the indicated treatment. qPCR analysis of mRNA of $A r$ in prostate tumors from representative mice used in the castration-naive (C) and castration-resistant (D) prostate cancer drug intervention trials in Figure 8. (E) qPCR analysis of mRNA of core AR-responsive genes in prostate tumors from C. Horizontal bars represent mean \pm SEM, and diamonds represent individual samples; $n=4$ mice/group. Significance represent Student-Newman-Keuls post hoc test for individual comparisons, upon significant 1-way ANOVA. Ar mRNA levels are shown in C. Tmprss2 and KIk4 were statistically significant for 1-way ANOVA and could not proceed to post hoc individual comparison. One-way ANOVA $P$ values are listed on the plot. ${ }^{*} P<0.05 ;{ }^{* *} P<0.01 ;{ }^{* *} P<0.001$.

We next explored whether the treatment combination of ISIS581088 and AZD5363 could enhance the therapeutic responses in a mouse model of advanced-stage CRPC. In this model, DKO mice with palpable prostate tumors $(0.5 \mathrm{~cm}$ maximum diameter $)$ were surgically castrated and randomized to treatment 2 weeks after orchidectomy or when tumors grow to $1 \mathrm{~cm}$ in diameter - whichever occurred sooner (Figure 10C). In this setting, median OS after randomization was 42 days in mice receiving ISIS581088 either alone or in combination with AZD5363 vs. 18 and 22 days in control vehicle and Ctrl-ASO mice, respectively (log-rank test, $P=0.258$, Supplemental Figure $8 \mathrm{C}$ ). Time to tumor progression (TTP) is used as a surrogate 
A

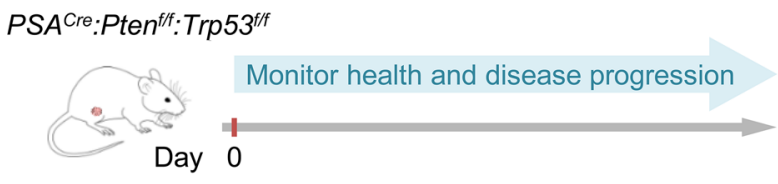

Randomize to treatment tumor when prostate tumor reach $1.0 \mathrm{~cm}$

C

PSA ${ }^{\text {Cre }}:$ Pten $^{\text {fft }}:$ Trp $53^{\text {ft }}$

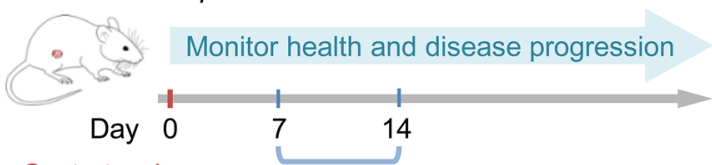

Castrate when prostate tumor reach $0.5 \mathrm{~cm}$

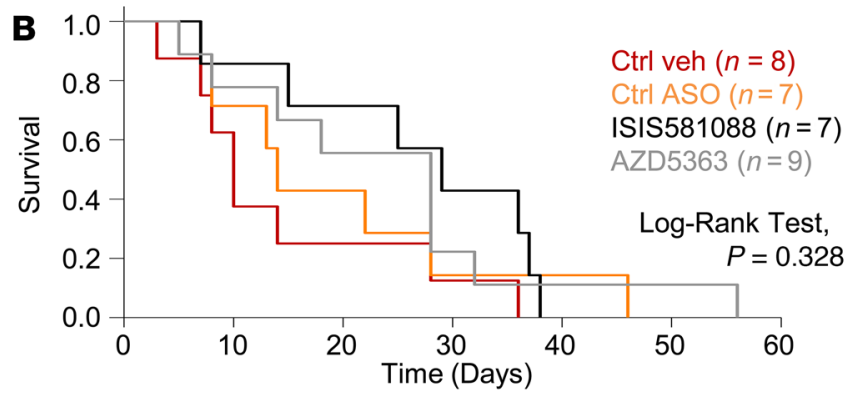

D

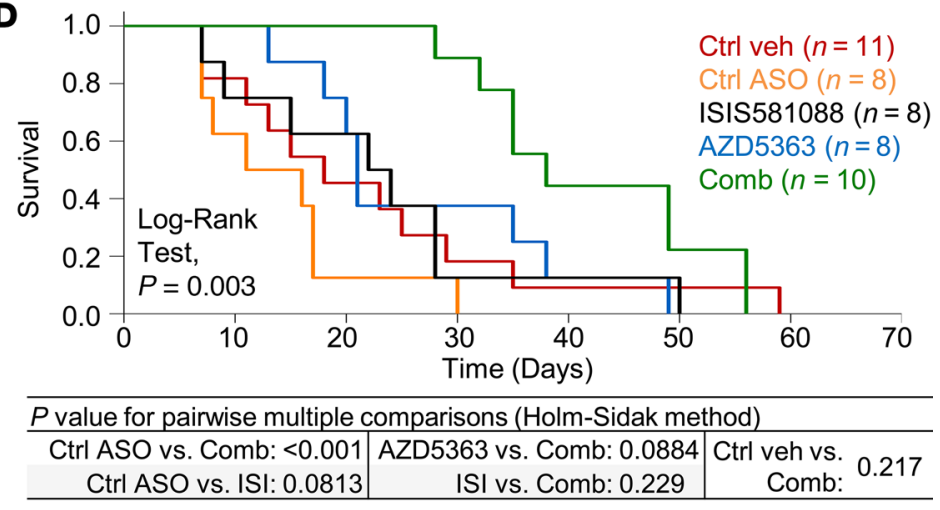

E

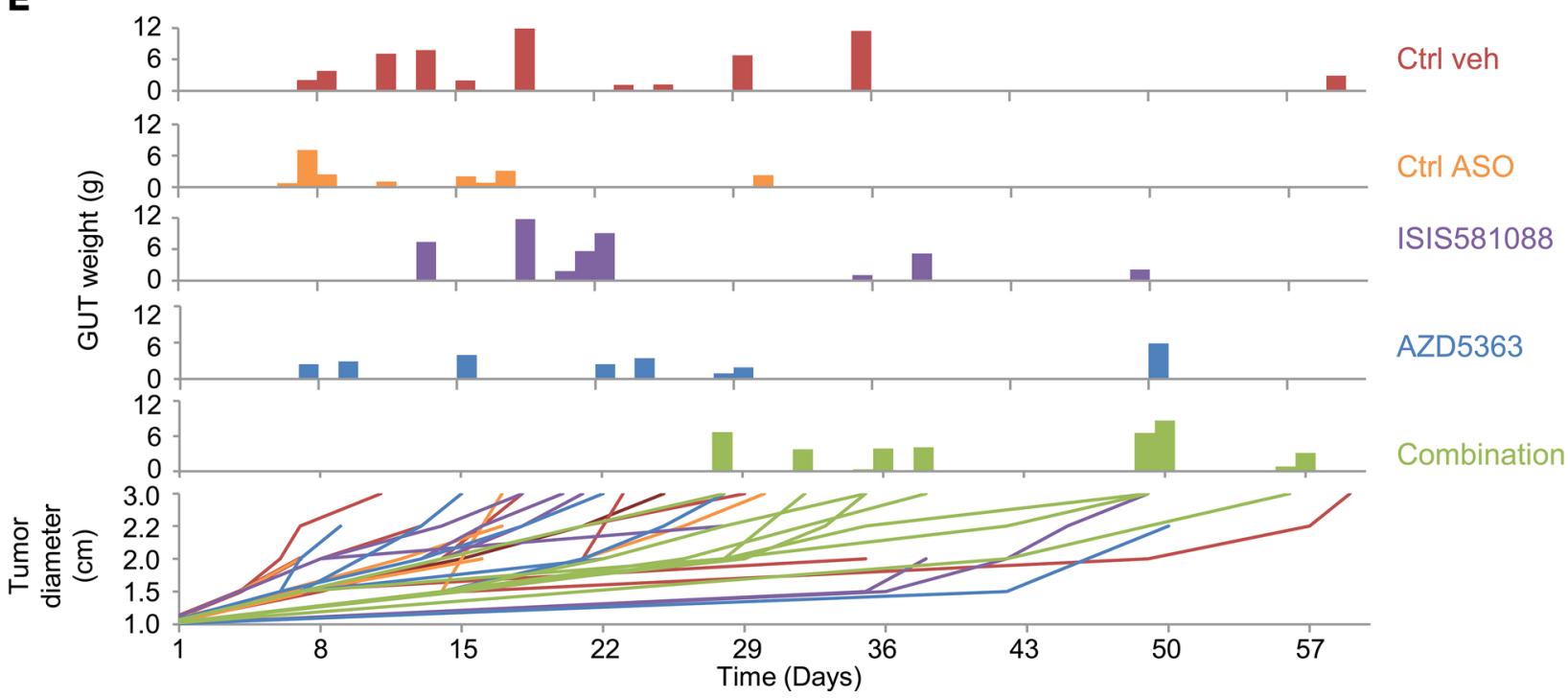

Figure 10. Combined AR and PI3K/AKT blockade suppresses cancer growth and improves survival in a mouse model of advanced castration-resistant prostate cancer. Experimental design for mouse survival studies using prostate-specific Pten/Trp53 conditional double KO mice in castration-naive (A) and castration-resistant prostate cancer (CRPC) (C) settings. Doses and treatment schedules were previously described (see Figure 5A). Kaplan-Meier plots showing overall survival curves for mice after the indicated treatment in castration-naive (B) and CRPC (D) settings. (E) Plots showing tumor burden relative to survival time and tumor growth curves.

marker for castration resistance in this model. Overall median times of TTP did not differ greatly between the groups; however, times varied widely, highlighting the heterogeneity of CRPC (Supplemental Figure $8 \mathrm{~B})$. To determine treatment response in a setting of CRPC, we measured OS after the tumors became castration resistant. In this context, the ISIS581088/AZD5363 treatment combination demonstrated a distinct advantage in OS compared with the other treatment groups (Figure 10D). Mice receiving combination therapy also demonstrated longer times to tumor growth and slower tumor growth curves, indicating tumor stabilization during treatment (Figure 10E and Supplemental Figure 8D). These findings demonstrate that combination therapy targeting both $\mathrm{AR}$ and AKT signaling is required in order to induce therapeutic responses in a model of advanced CRPC and provide preclinical evidence to support that a combination 
strategy using ASO targeting $A R$ in combination with AKT kinase inhibitors is a promising approach for the treatment of PTEN-deficient CRPC.

\section{Discussion}

For over 3 quarters of a century, blockade of AR signaling has been continuously regarded as a key objective for the management of advanced PCa. During this period, treatments have evolved from initial androgen ablation via surgical castration to pharmacological castration (luteinizing hormone-releasing hormone [LHRH] agonists), to contemporary strategies focusing more on AR blockade with antiandrogens such AR antagonists and androgen synthesis inhibitors. The second-generation antiandrogens enzalutamide and abiraterone have changed the therapeutic landscape for men suffering with CRPC by substantially improving survival in both chemotherapy-naive and postchemotherapy settings $(4-6,41)$. Despite these gains, some patients fail to respond, while others continue to eventually develop secondary resistance. Most of the mechanisms identified in enzalutamide and abiraterone resistance thus far implicate aberrant AR, which may result from $A R$ mutations and/or alternatively spliced AR variants (7, 42-45). Innovative programs are being carried out to identify druggable AR targets to screen and develop novel treatment compounds (46-48). Current potentially novel approaches that may curtail AR resistance such as those targeting the NTD of AR are being investigated as potential therapeutic options for CRPC (49).

CRPC is highly heterogeneous and most likely involves more than one mechanism of AR resistance. From this point of view, elimination of AR is a more appealing and potentially advantageous paradigm to treat AR-dependent cancer. Having the ability to eliminate all forms of AR would, in theory, provide a significant advantage to overcome many of the AR-dependent mechanisms that drive CRPC. Herein, we used ISIS581088, an ASO with a Generation-2.5 chemistry that improves stability and target affinity, to silence $A R$ gene expression in mouse Pten-deficient tumors and demonstrate that abrogation of AR can curtail AR-dependent prostate tumor growth. Chemical modifications influence the pharmacokinetic/pharmacodynamic properties of ASOs, extending their half-life and enhancing target specificity. First-generation ASOs used a phosphorothioate modification that improved cell permeability over unmodified ASOs, while second-generation ASOs included modifications to the backbone that led to greater target specificity and slowed degradation. Generation-2.5 ASOs use the cEt modification, which provides greater potency and selectivity over Generation-2.0 ASOs (50). Our pharmacodynamic study, indeed, showed a grade-dependent uptake of both ISIS581088 and Ctrl-ASO. This phenomenon may have resulted from enhanced distribution and increased cellular trafficking in high-grade mPIN. Enhanced distribution of ASOs to tumors with leaky vasculatures (high grade) has been reported, and it is generally accepted that ASOs enter cells via endocytic trafficking, a complex process that is regulated by cell signaling and is intricately involved in cancer progression $(51,52)$.

The efficacy of Generation-2.5 ASOs targeting human $A R$ has also been demonstrated in cell-based and patient-derived xenografts (PDX) preclinical models of PCa (13). In this study, the authors showed that $A R$-ASO could target both AR-FL and AR-Vs to suppress the growth of enzalutamide-resistant PCa cells, including those overexpressing AR-V7, which is associated with enzalutamide and abiraterone resistance (7). Most AR-Vs lack the LBD and are frequently overexpressed in CRPC, though at lower ratios than AR-FL $(18,31)$. In this study, we show that PCa development increased variant AR expression in mouse prostate tumors, which continued to be expressed during the progression to castration-resistant disease. Others have shown similarly sized functional AR-Vs in mouse PCa models that lack the LBD and are structurally similar to human AR-V7 $(29,30)$. It has also been reported that constitutively expressed AR-Vs can dimerize with AR-FL to maintain AR transcription in an androgen-independent manner (29, 53). We also noted the presence of high molecular weight bands expressed in prostate tumors from intact and castrated mice. More importantly, expression of these bands was reduced after Ar silencing with ISIS581088. We speculate that these bands may represent AR homodimers and AR/AR-V heterodimers. Additional studies will be needed in order to characterize the structural and functional role of aberrant AR-Vs in this model. Nevertheless, our studies demonstrate that ISIS581088-silenced $\mathrm{Ar}$ mRNA translation, targeting intron 1, was capable of abrogating all forms of AR. Other approaches aiming to abolish AR employ the use of agents that promote AR protein degradation $(54,55)$. Galeterone, a CYP17 inhibitor that has also shown evidence of degradation of AR-FL and the AR-V7 splice variant, showed promise in preclinical and early clinical evaluation. However, it failed to meet its primary endpoint: the pivotal Phase III ARMOR3-SV trial (NCT02438007; https://clinicaltrials.gov), evaluating galeterone vs. 
enzalutamide in treatment-naive AR-V7-positive metastatic CRPC patients. Other AR-degrading agents have also shown some promise and are being evaluated in the early clinical phase (49).

This study demonstrates that $A R$ silencing can successfully inhibit AR expression and function to suppress Pten-deficient tumor growth and is comparable in potency to surgical castration. In the setting of mouse Pten-deficient PCa, $A R$ silencing is superior to the second-generation antiandrogens enzalutamide, apalutamide, and abiraterone with regards to suppressing tumor growth and AR transcriptional activity. Our study also revealed that tumors treated with enzalutamide and abiraterone failed to decrease AR transcriptional activity as a result of increased $\mathrm{Ar}$ gene expression, suggesting that certain treatments may promote AR-dependent resistance mechanisms contributing to therapeutic resistance after long-term ADT, an occurrence that could be overcome with $A R$-ASO therapy. We also show that in vivo silencing of AR can exert distinct patterns of inhibition of $A R$-regulated genes between Pten-deficient CNPC and CRPC mouse models. Although our gene expression analysis is incomplete, as it focuses on a limited set of target genes, it still provides a snapshot of AR-target gene regulation in CRPC. Further experiments will need to be carried out to determine the precise transcriptional signature in our model. Nevertheless, our findings support other reports of distinct signatures of AR transcriptional regulation in in vitro and in vivo PCa models, as well as in clinical cancer specimens $(35,37)$. In particular, a shift in distinct transcriptional signatures between AR-FL and AR-Vs has been detailed (35). In our model, we identified the increased presence of putative AR-Vs, in Pten-deficient prostate tumors as well as their constitutive expression during the progression to CRPC, which may have contributed to the differences in transcriptional programming of AR. However, is important keep in mind that these and other AR transcriptional targets are not exclusive to AR, and their regulation may not be wholly dependent on AR, as other regulatory factors such as endogenous ligands, cofactors, and gene regulatory elements could be involved.

Notably, neither surgical castration nor AR silencing was sufficient to significantly improve survival in a mouse model of advanced Pten/Trp53-deficeint (DKO) CNPC, nor did it improve survival in CRPC. A lack of therapeutic efficacy from AR-ASO treatments suggested that other resistance mechanisms were active. Prostate tumors are highly dependent on PI3K/AKT signaling for survival and cooperate with AR signaling to promote therapeutic escape $(10,56,57)$. We previously showed that blockade of AKT using the pan-AKT inhibitor AZD5363 could significantly improve survival of DKO mice with CNPC; however, this treatment was ineffective against CRPC (26). In this study, we combined Ar silencing with AZD5363 blockade to target both AR and PI3K/AKT and, indeed, the agents significantly inhibited tumor progression, resulting in an OS benefit after development of CRPC. This study supports other studies and emphasizes the importance of concomitant inhibition of $\mathrm{AR}$ and $\mathrm{AKT}$ to enhance antitumor responses of $\mathrm{PCa}$ $(23,24,58,59)$. Although we showed a significant improvement in treatment response, tumors invariably continued to grow, indicating that other mechanisms are being coordinated to promote tumor survival. Therefore, it will be critical to perform further analysis to identify which mechanisms are contributing to promote therapeutic escape.

Therapeutic ASOs have been approved or are in late-stage clinical development for several difficult-to-treat diseases. Nevertheless, obstacles have hindered their potential as effective therapeutic molecules. Large size and highly anionic structure have limited the ability of ASOs to maximize their full effective potential (60). Recent advances in therapeutic ASOs formulations have renewed interest as potential anticancer agents. Several Generation-2.5 ASOs have shown promise in preclinical cancer models and early-phase clinical trials $(13,50,61,62)$. AZD5312 (IONIS-ARRx), a Generation-2.5 ASO designed to target full-length, splice variant, and mutated forms of human AR, recently completed phase I evaluation and showed good safety and tolerability in heavily pretreated mCRPC patients (NCT02144051) (62).

In conclusion, we have demonstrated the potency of a Generation-2.5 ASO targeting Ar using in vivo GEM models of PCa and have shown that this therapeutic approach effectively downregulated $\mathrm{Ar}$ to suppress prostate tumor growth in both castration-naive and castration-resistant models. Our data also provide additional evidence to support reciprocal feedback regulation between AR and the PI3K/ AKT pathway. Most importantly, we demonstrated that a rational combinatorial approach using a next-generation ASO to silence Ar can cooperate with AKT inhibition to mitigate therapeutic escape of monotherapy in the setting of Pten-deficient prostate tumors. Together, these data provide compelling evidence for further investigation of the pharmacological blockade of AR with Generation-2.5 ASO and AKT inhibition for the treatment of CRPC. 


\section{Methods}

Treatment compounds. The sequences of the Generation-2.5 ASOs are 5'-CCGAATCATATCTGCC-3' for ISIS581088 and 5'-GGCTACTACGCCGTCA-3' for Ctrl-ASO. The Generation-2.5 ASOs were formulated in saline and were administered i.p. at the indicated doses and schedules. AZD5363 was provided by AstraZeneca and was dosed and prepared as previously described (26). Enzalutamide, apalutamide, and abiraterone acetate were purchased from MedChem Express and were prepared as previously described (2, 33, 63). CMA was a gift from ASKA Pharmaceutical Co. Ltd. and was prepared as previously described (64).

Mice and surgical manipulation. Pten-deficient $\left(P S A^{C r e} ; P t e n^{\text {loxP/loxP }}\right.$ on a C57BL/6J background) and DKO (PSACre:Pten ${ }^{\text {loxP/loxP }} / \operatorname{Trp} 3^{\text {loxP/loxP }}$ on a mixed C57BL/6J and FVB/N background) prostate GEM models were bred in our laboratory and have been described previously $(25,26)$. Founder $P S A^{C r e}$ and $P$ ten ${ }^{\text {loxP } / \text { loxP }}$ mice were provided by Jeffrey Milbrandt (Washington University, St. Louis, Missouri, USA) and Tak Mak (Ontario Cancer Institute/University of Toronto, Toronto, Canada), respectively. Trp53 ${ }^{\text {loxP } / \text { loxP }}$ mice (strain no. 01XC2) were obtained through the NCI Mouse Repository (http://frederick.cancer.gov/science/technology/mouserepository). Mice were orchidectomized as previously described (25).

In vitro studies. Murine PCa cell lines were established from Pten-deficient and DKO mice as previously described (65). Cell lines were maintained under standard cell culture conditions using 10\% FBS, 1\% penicillin/streptomycin (P/S) RPMI Media. Crystal violet staining was used to determine cell viability. The median effective dose $\left(\mathrm{ED}_{50}\right)$ for cell viability was determined by continuous exposure of the respective cells to ISIS581088 or AZD5363 (AstraZeneca) for 96 hours at the indicated doses. Drug combination effects on cell viability were performed using a constant-ratio design, and synergy was determined by the combination index (CI) method based on the median effect analysis according to the Chou-Talalay method. CI values were generated using CompuSyn Software (http://www.combosyn.com).

In vivo efficacy studies. Drug efficacy studies were performed on age-matched Pten-deficient mice with castration-naive or castration-resistant prostate tumors and were randomized (stratified random sampling method) to treatments as indicated. Treatment regimens, protocols, and dosages are detailed in Supplemental Table 4. Mice treated with ISIS581088 or Ctrl-ASO were euthanized 24 hours after the final treatment. For all other treatments, mice were euthanized 2 hours after the last administered dose. GUTs were removed, weighed, and imaged. The prostate glands were dissected out bilaterally portioned and were either flash frozen in liquid nitrogen, preserved in RNAlater solution (Invitrogen), or fixed overnight in 10\% neutral buffered formalin; processed; and embedded in paraffin for further analysis. Antitumor activity was determined by comparing prostate burden calculated by tumor surface area as previously described or prostate weight (25).

Mouse survival studies. Survival studies were conducted on DKO mice as previously described (26). Briefly, DKO mice were palpated and were surgically castrated when prostate tumor reached $0.5 \mathrm{~cm}$ in diameter. Mice were palpated weekly and randomized (permuted block randomization) to treatment cohorts after 2 weeks or when tumor size increased to $1 \mathrm{~cm}$ - whichever occurred first. Mice were monitored daily, and tumors were palpated weekly. Mice were sacrificed when tumors reached $3 \mathrm{~cm}$ in maximum diameter or when miceexperienced $>20 \%$ body weight loss from baseline, poor performance status, abnormal behavior/ demeanor, distended abdomen, or hematuria. Performance status of mice was used to quantify the overall well-being of tumor-bearing mice receiving therapy. Mice were assigned a score ranging from $0-4$ as follows: 0 , asymptomatic; 1 , mildly symptomatic; 2 , symptomatic; 3 , dead. A favorable status was allocated to mice with a performance score $\leq 1$, and a poor status to those with a score $\geq 2$. Primary endpoints were safety and drug tolerability, OS, and TTP (defined as initial tumor doubling time). Secondary endpoints were primary tumor burden (weight of GUT), metastatic disease (presence and number of gross lesions), tumor growth rates, and overall health (performance status).

Histopathology and IHC analysis. For histological analysis, formalin-fixed paraffin embedded (FFPE) prostates were sectioned and stained with H\&E. For IHC studies, FFPE tissue sections were sectioned and placed on positively charged slides and incubated with the primary antibody, stained using the $\mathrm{ABC}$ kit (Vector Laboratories) following manufacturer's protocols, developed in DAB (Invitrogen), and counterstained with hematoxylin. Primary antibodies and specific pretreatments are listed in Supplemental Table 7. Histology slides were reviewed by a veterinary pathologist or trained research staff. Classification of mouse PIN lesions were carried out according to the guidelines previously published (66). Assessment of IHC staining was performed on whole prostates sections captured at $10 \times$ magnification and stitched with Adobe Photoshop CS5 Extended version 12.0.4; positivity scores were calculated using Aperio's Image Scope image analysis software version 12.3.2 using the Positive Pixel Count v9 algorithm. 
Western blotting and capillary electrophoresis. Protein extraction was performed in snap-frozen prostate tumors using RIPA buffer with HALT protease and phosphatase inhibitors (Thermo Fisher Scientific). SDSgel electrophoresis was carried out on Novex 4-20\% Tris-Glycine Gels (Invitrogen), transferred to Immobilon-P PVDF membranes (MilliporeSigma), and blocked with 3\% BSA-TBST (50 mM Tris-HCl, $150 \mathrm{mM}$ $\mathrm{NaCl}$, Tween-20; MilliporeSigma) before probing with primary antibodies listed in Supplemental Table 5. Blots were developed in ECL Prime solution (GE Healthcare) to detect bands, and the images were captured using the LAS-4010 ImageQuant imaging system (GE Healthcare). Membranes were stripped by incubating them in SDS plus $\beta$-mercaptoethanol solution (62.5 $\mathrm{mM}$ tris [pH 6.8], 2\% SDS [MilliporeSigma], and 100 $\mathrm{mM} \beta$-mercaptoethanol [Bio-Rad]) and incubated at $50^{\circ} \mathrm{C}$ for 30 minutes. Stripped membranes were washed in TBST buffer, blocked, and reprobed with additional primary antibodies as required. Semiquantitative densitometric analyses were performed using ImageJ (NIH) analysis software (http://imagej.nih.gov/ij). Capillary electrophoresis was performed using a Wes automated system (ProteinSimple) according to the manufacturer's instructions. Purified recombinant proteins were used as calibration standards. Digital Western blot analyses were performed with Compass version 2.7.1 analysis software.

$R N A$ extraction and expression analysis. Total RNA was extracted and purified from prostate tissues preserved in RNAlater solution using the RNeasy Mini Kit (Qiagen) following the manufacturer's instructions. cDNA was synthesized using the PrimeScript First Strand cDNA Synthesis Kit (Takara) according to the manufacturer's protocol. Primers for genes used in the analyses are shown in Supplemental Table 8 and were designed using Primer-Blast or obtained from the qPrimerDepot database (67). cDNA concentration was measured by qPCR using the SYBR Premix Ex Taq II Kit (Takara). Relative change in gene expression was calculated using $2^{-\Delta \Delta C T}$ method using $G A P D H$ as an internal control. qPCR arrays were performed on the Fluidigm Biomark platform using a panel of 86 mouse $A r$-responsive genes extracted from the literature and reported to be AR-responsive in mice (23). Reverse transcription was performed with $50 \mathrm{ng}$ of total RNA in a final volume of $20 \mu \mathrm{l}$, using the High Capacity cDNA Reverse Transcription kit (Invitrogen). The following thermal profile was used in the reaction: $25^{\circ} \mathrm{C}$ for 10 minutes, $37^{\circ} \mathrm{C}$ for 120 minutes, $85^{\circ} \mathrm{C}$ for 5 seconds, and $4^{\circ} \mathrm{C}$ for 2 minutes. Preamplification was performed with $1.25 \mu \mathrm{l}$ of resulting cDNA in a final volume of $5 \mu 1$, using a pool of TaqMan assays at a final dilution of 1 in 100 and TaqMan PreAmp Master Mix (Invitrogen). The following thermal profile was used in the reaction: $95^{\circ} \mathrm{C}$ for 10 minutes, 14 cycles of $95^{\circ} \mathrm{C}$ for 15 seconds, and $60^{\circ} \mathrm{C}$ for 4 minutes. Preamplified samples were diluted 1 in 5 with Tris-EDTA (TE) buffer prior to analysis on the Fluidigm BioMark System. Sample and assay preparation for Fluidigm Gene Expression Dynamic Arrays were performed according to manufacturer's instructions. Briefly, samples were mixed with GE Sample Loading Reagent (Fluidigm) and TaqMan Gene Expression Master Mix (Applied Biosystems). Assays were mixed with GE Assay Loading Reagent (Fluidigm). The Fluidigm Dynamic Arrays were primed and loaded on an IFC Controller (Fluidigm), and qPCR was performed on a BioMark System (Fluidigm) using the following thermal profile: $50^{\circ} \mathrm{C}$ for 2 minutes, $70^{\circ} \mathrm{C}$ for 30 minutes, $25^{\circ} \mathrm{C}$ for 10 minutes, $50^{\circ} \mathrm{C}$ for 2 minutes, and $95^{\circ} \mathrm{C}$ for 10 minutes, as well as 40 cycles of $95^{\circ} \mathrm{C}$ for 15 seconds and $60^{\circ} \mathrm{C}$ for 1 minute. Data was analyzed using the Fluidigm BioMark Real-Time PCR Analysis software version 3.1.3, and gene expression values were calculated using the $\Delta \Delta \mathrm{Ct}$ method.

Network analysis. FunRich and ToppCluster were used to perform enrichment and functional network analyses $(68,69)$.

Statistics. Statistical analyses were performed with Sigmaplot 13 and $\mathrm{MeV}$ software. Hierarchical cluster, corresponding statistical analysis, and heatmaps were performed with the TM4-MeV v4.9.0 software application (http://mev.tm4.org). A 2-tailed Student's $t$ test was used to compare differences between 2 groups and 1-way ANOVA (Student-Newman-Keuls Method) or 1-way ANOVA on ranks (Dunn's Kruskal-Wallis Multiple Comparisons) were used to compare differences between multiple groups. Correlation analysis was performed with Past-v3.2 software (http://folk.uio.no/ohammer/past). $P<0.05$ was considered significant.

Study approval. Mice were housed at the Kindai University Faculty of Medicine Animal Facility in accordance with institutional guidelines, and procedures were carried out in compliance with the standards for use of laboratory animals.

\section{Author contributions}

MAD and HU conceived and designed the project. MAD, YK, K. Sugimoto, and YH acquired the data. MAD and HU developed methodology. MAD, K. Sakai, K. Sugimoto, HC, and SK performed data analysis. BRD, YK, and ARM provided administrative, technical, or material support. KY and KN provided expert consultation. MAD, KN, and HU supervised the study. MAD wrote the manuscript with input from all authors 


\section{Acknowledgments}

Research grant support was provided by the Japan Society for the Promotion of Science and AstraZeneca. AZD5363/capivasertib was discovered by AstraZeneca subsequent to a collaboration with Astex Therapeutics (and its collaboration with the Institute of Cancer Research and Cancer Research Technology Limited)

Address correspondence to: Marco A. De Velasco, Department of Genome Biology, Kindai University Faculty of Medicine, 377-2 Ohno-Higashi, Osakasayama, Osaka, Japan 589-8511. Phone: 81.72.366.0221 ext. 3523; Email: mdev@med.kindai.ac.jp. Or to: Hirotsugu Uemura, Department of Urology, Kindai University Faculty of Medicine, 377-2 Ohno-Higashi, Osakasayama, Osaka, Japan 589-8511. Phone: 81.72.366.0221 ext.3524; Email: huemura@med.kindai.ac.jp.

1. Zhou Y, Bolton EC, Jones JO. Androgens and androgen receptor signaling in prostate tumorigenesis. J Mol Endocrinol. 2015;54(1):R15-R29.

2. Tran C, et al. Development of a second-generation antiandrogen for treatment of advanced prostate cancer. Science. 2009;324(5928):787-790.

3. Attard G, Belldegrun AS, de Bono JS. Selective blockade of androgenic steroid synthesis by novel lyase inhibitors as a therapeutic strategy for treating metastatic prostate cancer. BJU Int. 2005;96(9):1241-1246.

4. Penson DF, et al. Enzalutamide Versus Bicalutamide in Castration-Resistant Prostate Cancer: The STRIVE Trial. J Clin Oncol. 2016;34(18):2098-2106.

5. Scher HI, et al. Increased survival with enzalutamide in prostate cancer after chemotherapy. $N$ Engl J Med. 2012;367(13):1187-1197.

6. de Bono JS, et al. Abiraterone and increased survival in metastatic prostate cancer. N Engl J Med. 2011;364(21):1995-2005

7. Antonarakis ES, et al. AR-V7 and resistance to enzalutamide and abiraterone in prostate cancer. N Engl J Med. 2014;371(11):1028-1038.

8. Bubley GJ, Balk SP. Association Between Androgen Receptor Splice Variants and Prostate Cancer Resistance to Abiraterone and Enzalutamide. J Clin Oncol. 2017;35(19):2103-2105.

9. Kaarbø M, Klokk TI, Saatcioglu F. Androgen signaling and its interactions with other signaling pathways in prostate cancer. Bioessays. 2007;29(12):1227-1238.

10. Kahn B, Collazo J, Kyprianou N. Androgen receptor as a driver of therapeutic resistance in advanced prostate cancer. Int J Biol Sci. 2014;10(6):588-595.

11. Sun S, et al. Castration resistance in human prostate cancer is conferred by a frequently occurring androgen receptor splice variant. J Clin Invest. 2010;120(8):2715-2730.

12. Hung G, et al. Characterization of target mRNA reduction through in situ RNA hybridization in multiple organ systems following systemic antisense treatment in animals. Nucleic Acid Ther. 2013;23(6):369-378.

13. Yamamoto Y, et al. Generation 2.5 antisense oligonucleotides targeting the androgen receptor and its splice variants suppress enzalutamide-resistant prostate cancer cell growth. Clin Cancer Res. 2015;21(7):1675-1687.

14. Davies MA. Regulation, role, and targeting of Akt in cancer. J Clin Oncol. 2011;29(35):4715-4717.

15. Hemmings BA, Restuccia DF. PI3K-PKB/Akt pathway. Cold Spring Harb Perspect Biol. 2012;4(9):a011189.

16. Barbieri CE, et al. The mutational landscape of prostate cancer. Eur Urol. 2013;64(4):567-576.

17. Grasso CS, et al. The mutational landscape of lethal castration-resistant prostate cancer. Nature. 2012;487(7406):239-243.

18. Drake JM, et al. Metastatic castration-resistant prostate cancer reveals intrapatient similarity and interpatient heterogeneity of therapeutic kinase targets. Proc Natl Acad Sci USA. 2013;110(49):E4762-E4769.

19. Robinson D, et al. Integrative clinical genomics of advanced prostate cancer. Cell. 2015;161(5):1215-1228

20. Taylor BS, et al. Integrative genomic profiling of human prostate cancer. Cancer Cell. 2010;18(1):11-22.

21. Di Lorenzo G, et al. Expression of epidermal growth factor receptor correlates with disease relapse and progression to androgen-independence in human prostate cancer. Clin Cancer Res. 2002;8(11):3438-3444.

22. De Velasco MA, Uemura H. Preclinical Remodeling of Human Prostate Cancer through the PTEN/AKT Pathway. Adv Urol. 2012;2012:419348.

23. Carver BS, et al. Reciprocal feedback regulation of PI3K and androgen receptor signaling in PTEN-deficient prostate cancer. Cancer Cell. 2011;19(5):575-586.

24. Mulholland DJ, et al. Cell autonomous role of PTEN in regulating castration-resistant prostate cancer growth. Cancer Cell. 2011;19(6):792-804.

25. De Velasco MA, et al. Androgen deprivation induces phenotypic plasticity and promotes resistance to molecular targeted therapy in a PTEN-deficient mouse model of prostate cancer. Carcinogenesis. 2014;35(9):2142-2153.

26. De Velasco MA, Kura Y, Yoshikawa K, Nishio K, Davies BR, Uemura H. Efficacy of targeted AKT inhibition in genetically engineered mouse models of PTEN-deficient prostate cancer. Oncotarget. 2016;7(13):15959-15976.

27. Talbot DC, et al. Tumor survivin is downregulated by the antisense oligonucleotide LY2181308: a proof-of-concept, first-inhuman dose study. Clin Cancer Res. 2010;16(24):6150-6158.

28. Li Y, Chan SC, Brand LJ, Hwang TH, Silverstein KA, Dehm SM. Androgen receptor splice variants mediate enzalutamide resistance in castration-resistant prostate cancer cell lines. Cancer Res. 2013;73(2):483-489.

29. Watson PA, et al. Constitutively active androgen receptor splice variants expressed in castration-resistant prostate cancer require full-length androgen receptor. Proc Natl Acad Sci USA. 2010;107(39):16759-16765. 
30. Liang M, et al. Identification of Androgen Receptor Splice Variants in the Pten Deficient Murine Prostate Cancer Model. PLoS ONE. 2015;10(7):e0131232.

31. Wadosky KM, Koochekpour S. Androgen receptor splice variants and prostate cancer: From bench to bedside. Oncotarget. 2017;8(11):18550-18576.

32. Shafi AA, Cox MB, Weigel NL. Androgen receptor splice variants are resistant to inhibitors of Hsp90 and FKBP52, which alter androgen receptor activity and expression. Steroids. 2013;78(6):548-554.

33. Clegg NJ, et al. ARN-509: a novel antiandrogen for prostate cancer treatment. Cancer Res. 2012;72(6):1494-1503.

34. Térouanne B, Paris F, Servant N, Georget V, Sultan C. Evidence that chlormadinone acetate exhibits antiandrogenic activity in androgen-dependent cell line. Mol Cell Endocrinol. 2002;198(1-2):143-147.

35. Hu R, et al. Distinct transcriptional programs mediated by the ligand-dependent full-length androgen receptor and its splice variants in castration-resistant prostate cancer. Cancer Res. 2012;72(14):3457-3462.

36. Lonergan PE, Tindall DJ. Androgen receptor signaling in prostate cancer development and progression. J Carcinog. 2011;10:20.

37. Sharma NL, et al. The androgen receptor induces a distinct transcriptional program in castration-resistant prostate cancer in man. Cancer Cell. 2013;23(1):35-47.

38. Dhiman VK, Attwood K, Campbell MJ, Smiraglia DJ. Hormone stimulation of androgen receptor mediates dynamic changes in DNA methylation patterns at regulatory elements. Oncotarget. 2015;6(40):42575-42589.

39. Ho ME, et al. Prostate cancer cell phenotypes based on AGR2 and CD10 expression. Mod Pathol. 2013;26(6):849-859.

40. Dall'Era MA, True LD, Siegel AF, Porter MP, Sherertz TM, Liu AY. Differential expression of CD10 in prostate cancer and its clinical implication. BMC Urol. 2007;7:3.

41. Ryan CJ, et al. Abiraterone acetate plus prednisone versus placebo plus prednisone in chemotherapy-naive men with metastatic castration-resistant prostate cancer (COU-AA-302): final overall survival analysis of a randomised, double-blind, placebo-controlled phase 3 study. Lancet Oncol. 2015;16(2):152-160.

42. Azad AA, et al. Androgen Receptor Gene Aberrations in Circulating Cell-Free DNA: Biomarkers of Therapeutic Resistance in Castration-Resistant Prostate Cancer. Clin Cancer Res. 2015;21(10):2315-2324.

43. Joseph JD, et al. A clinically relevant androgen receptor mutation confers resistance to second-generation antiandrogens enzalutamide and ARN-509. Cancer Discov. 2013;3(9):1020-1029.

44. Korpal M, et al. An F876L mutation in androgen receptor confers genetic and phenotypic resistance to MDV3100 (enzalutamide) Cancer Discov. 2013;3(9):1030-1043.

45. Romanel A, et al. Plasma AR and abiraterone-resistant prostate cancer. Sci Transl Med. 2015;7(312):312re10.

46. Shen HC, et al. In silico discovery of androgen receptor antagonists with activity in castration resistant prostate cancer. $M o l$ Endocrinol. 2012;26(11):1836-1846.

47. Balbas MD, et al. Overcoming mutation-based resistance to antiandrogens with rational drug design. Elife. 2013;2:e00499.

48. Munuganti RS, et al. Targeting the binding function 3 (BF3) site of the androgen receptor through virtual screening. 2 development of 2-((2-phenoxyethyl) thio)-1H-benzimidazole derivatives. J Med Chem. 2013;56(3):1136-1148.

49. Antonarakis ES, Chandhasin C, Osbourne E, Luo J, Sadar MD, Perabo F. Targeting the N-Terminal Domain of the Androgen Receptor: A New Approach for the Treatment of Advanced Prostate Cancer. Oncologist. 2016;21(12):1427-1435.

50. Hong D, et al. AZD9150, a next-generation antisense oligonucleotide inhibitor of STAT3 with early evidence of clinical activity in lymphoma and lung cancer. Sci Transl Med. 2015;7(314):314ra185.

51. Geary RS, Norris D, Yu R, Bennett CF. Pharmacokinetics, biodistribution and cell uptake of antisense oligonucleotides. Adv Drug Deliv Rev. 2015;87:46-51.

52. Schmid SL. Reciprocal regulation of signaling and endocytosis: Implications for the evolving cancer cell. J Cell Biol. 2017;216(9):2623-2632.

53. Xu D, et al. Androgen Receptor Splice Variants Dimerize to Transactivate Target Genes. Cancer Res. 2015;75(17):3663-3671

54. Wang R, Lin W, Lin C, Li L, Sun Y, Chang C. ASC-J9(®) suppresses castration resistant prostate cancer progression via degrading the enzalutamide-induced androgen receptor mutant AR-F876L. Cancer Lett. 2016;379(1):154-160.

55. Yu Z, Cai C, Gao S, Simon NI, Shen HC, Balk SP. Galeterone prevents androgen receptor binding to chromatin and enhances degradation of mutant androgen receptor. Clin Cancer Res. 2014;20(15):4075-4085

56. Lee SH, Johnson D, Luong R, Sun Z. Crosstalking between androgen and PI3K/AKT signaling pathways in prostate cancer cells. J Biol Chem. 2015;290(5):2759-2768.

57. Crumbaker M, Khoja L, Joshua AM. AR Signaling and the PI3K Pathway in Prostate Cancer. Cancers (Basel). $2017 ; 9(4)$ :E34.

58. Yadav SS, et al. Combination effect of therapies targeting the PI3K- and AR-signaling pathways in prostate cancer. Oncotarget. 2016;7(46):76181-76196.

59. Marques RB, et al. High Efficacy of Combination Therapy Using PI3K/AKT Inhibitors with Androgen Deprivation in Prostate Cancer Preclinical Models. Eur Urol. 2015;67(6):1177-1185.

60. Khvorova A, Watts JK. The chemical evolution of oligonucleotide therapies of clinical utility. Nat Biotechnol. 2017;35(3):238-248

61. Ross SJ, et al. Targeting KRAS-dependent tumors with AZD4785, a high-affinity therapeutic antisense oligonucleotide inhibitor of KRAS. Sci Transl Med. 2017;9(394):eaal5253.

62. Chowdhury S, et al. A phase I dose escalation, safety and pharmacokinetic (PK) study of AZD5312 (IONIS-ARRx), a first-inclass Generation 2.5 antisense oligonucleotide targeting the androgen receptor (AR). Eur J Cancer. 2016;69(S1):S145.

63. Li R, et al. Abiraterone inhibits 3 $\beta$-hydroxysteroid dehydrogenase: a rationale for increasing drug exposure in castration-resistant prostate cancer. Clin Cancer Res. 2012;18(13):3571-3579.

64. Koike H, et al. Conditional PTEN-deficient mice as a prostate cancer chemoprevention model. Asian Pac J Cancer Prev. 2015;16(5):1827-1831

65. Takao A, et al. Generation of PTEN-knockout (-/-) murine prostate cancer cells using the CRISPR/Cas9 system and comprehensive gene expression profiling. Oncol Rep. 2018;40(5):2455-2466.

66. Ittmann M, et al. Animal models of human prostate cancer: the consensus report of the New York meeting of the Mouse Models of Human Cancers Consortium Prostate Pathology Committee. Cancer Res. 2013;73(9):2718-2736.

67. Cui W, Taub DD, Gardner K. qPrimerDepot: a primer database for quantitative real time PCR. Nucleic Acids Res. 2007;35(Database 
issue):D805-D809.

68. Pathan M, et al. FunRich: An open access standalone functional enrichment and interaction network analysis tool. Proteomics. 2015;15(15):2597-2601.

69. Kaimal V, Bardes EE, Tabar SC, Jegga AG, Aronow BJ. ToppCluster: a multiple gene list feature analyzer for comparative enrichment clustering and network-based dissection of biological systems. Nucleic Acids Res. 2010;38(Web Server issue):W96-102. 